\title{
NUMERICAL COMPUTATION OF DEFLATING SUBSPACES OF SKEW-HAMILTONIAN/HAMILTONIAN PENCILS*
}

\author{
PETER BENNER ${ }^{\dagger}$, RALPH BYERS $\ddagger$, VOLKER MEHRMANN ${ }^{\S}$, AND HONGGUO XUף
}

\begin{abstract}
We discuss the numerical solution of structured generalized eigenvalue problems that arise from linear-quadratic optimal control problems, $H_{\infty}$ optimization, multibody systems, and many other areas of applied mathematics, physics, and chemistry. The classical approach for these problems requires computing invariant and deflating subspaces of matrices and matrix pencils with Hamiltonian and/or skew-Hamiltonian structure. We extend the recently developed methods for Hamiltonian matrices to the general case of skew-Hamiltonian/Hamiltonian pencils. The algorithms circumvent problems with skew-Hamiltonian/Hamiltonian matrix pencils that lack structured Schur forms by embedding them into matrix pencils that always admit a structured Schur form. The rounding error analysis of the resulting algorithms is favorable. For the embedded matrix pencils, the algorithms use structure preserving unitary matrix computations and are strongly backwards stable, i.e., they compute the exact structured Schur form of a nearby matrix pencil with the same structure.
\end{abstract}

Keywords. eigenvalue problem, deflating subspace, Hamiltonian matrix, skew-Hamiltonian matrix, skew-Hamiltonian/Hamiltonian matrix pencil.

AMS subject classification. 49N10, 65F15, 93B40, 93B36.

1. Introduction and Preliminaries. In this paper we study eigenvalue and invariant subspace computations involving matrices and matrix pencils with the following algebraic structures.

Definition 1.1. Let $\mathcal{J}:=\left[\begin{array}{cc}0 & I_{n} \\ -I_{n} & 0\end{array}\right]$, where $I_{n}$ is the $n \times n$ identity matrix.

a) A matrix $\mathcal{H} \in \mathbb{C}^{2 n, 2 n}$ is Hamiltonian if $(\mathcal{H} \mathcal{J})^{H}=\mathcal{H} \mathcal{J}$. The Lie Algebra of Hamiltonian matrices in $\mathbb{C}^{2 n, 2 n}$ is denoted by $\mathbb{H}_{2 n}$.

b) A matrix $\mathcal{H} \in \mathbb{C}^{2 n, 2 n}$ is skew-Hamiltonian if $(\mathcal{H} \mathcal{J})^{H}=-\mathcal{H} \mathcal{J}$. The Jordan algebra of skew-Hamiltonian matrices in $\mathbb{C}^{2 n, 2 n}$ is denoted by $\mathbb{S H}_{2 n}$.

c) If $\mathcal{S} \in \mathbb{S H}_{2 n}$ and $\mathcal{H} \in \mathbb{H}_{2 n}$, then $\alpha \mathcal{S}-\beta \mathcal{H}$ is a skew-Hamiltonian/Hamiltonian matrix pencil.

d) A matrix $\mathcal{Y} \in \mathbb{C}^{2 n, 2 n}$ is symplectic if $\mathcal{Y} \mathcal{J} \mathcal{Y}^{H}=\mathcal{J}$. The Lie group of symplectic matrices in $\mathbb{C}^{2 n, 2 n}$ is denoted by $\mathbb{S}_{2 n}$.

e) A matrix $\mathcal{U} \in \mathbb{C}^{2 n, 2 n}$ is unitary symplectic if $\mathcal{U} \mathcal{J U}^{H}=\mathcal{J}$ and $\mathcal{U U}^{H}=I_{2 n}$. The compact Lie group of unitary symplectic matrices in $\mathbb{C}^{2 n, 2 n}$ is denoted by $\mathbb{U S}_{2 n}$.

f) A subspace $\mathcal{L}$ of $\mathbb{C}^{2 n}$ is called Lagrangian if it has dimension $n$ and $x^{H} \mathcal{J} y=0$ for all $x, y \in \mathcal{L}$.

A matrix $\mathcal{S} \in \mathbb{C}^{2 n, 2 n}$ is skew-Hamiltonian if and only if $i \mathcal{S}$ is Hamiltonian. Consequently, there is little difference between the structure of complex skew-Hamiltonian matrices and complex Hamiltonian matrices. However, real skew-Hamiltonian matrices are not real scalar multiples of Hamiltonian matrices, so there is a greater difference between the structure of real skew-Hamiltonian matrices and real Hamiltonian matrices.

The structures in Definition 1.1 arise typically in linear-quadratic optimal control [27, 33, 35] and $H_{\infty}$ optimization $[18,39]$. Moreover, instances of skew-Hamiltonian/Hamiltonian pencils appear in several other areas of applied mathematics, computational physics and chemistry, e.g., gyroscopic systems [20], numerical simulation of elastic deformation [28, 34], and linear response

*Working title was "Numerical Computation of Deflating Subspaces of Embedded Hamiltonian and Symplectic Pencils". All authors were partially supported by Deutsche Forschungsgemeinschaft, Research Grant Me 790/7-2 and Sonderforschungsbereich 393, "Numerische Simulation auf massiv parallelen Rechnern".

$\dagger$ Zentrum für Technomathematik, Fachbereich 3/Mathematik und Informatik, Universität Bremen, D-28334 Bremen, Germany, benner@math.uni-bremen.de.

$\ddagger$ Department of Mathematics, University of Kansas, Lawrence, KS 66045, USA, byers@math.ukans.edu. This author was partially supported by National Science Foundation awards CCR-9732671, MRI-9977352, and by the NSF EPSCoR/K*STAR program through the Center for Advanced Scientific Computing.

$\S$ Fachbereich 3 Mathematik, Technische Universität Berlin, Sekr. MA 4-5, Str. des 17. Juni 136, D-10623 Berlin, Germany, mehrmann@math.tu-berlin.de

I Deparment of Mathematics, University of Kansas, Lawrence, KS 66045, USA, xu@math.ukans.edu. This work was completed while this author was with the TU Chemnitz, Germany. 
theory [30]. Linear-quadratic optimal control and $H_{\infty}$ optimization problems are related to skewHamiltonian/Hamiltonian pencils in $[4,5]$.

It is important to exploit and preserve algebraic structures (like symmetries in the matrix blocks or symmetries in the spectrum) as much as possible. Such algebraic structures typically arise from the physical properties of the problem. If rounding errors or other perturbations destroy the algebraic structures, then the results may be physically meaningless. Not coincidentally, numerical methods that preserve algebraic structures are typically more efficient as well as more accurate.

Despite the advantages associated with exploiting matrices with special structure, condensing data into a compact, structured matrix using finite precision arithmetic may be ill-advised. A discussion of avoiding normal-equations-like numerical instability when embedding linear-quadratic optimal control problems and $H_{\infty}$ optimization problems into skew-Hamiltonian/Hamiltonian pencils appears in $[4,5]$.

Although the numerical computation of $n$-dimensional Lagrangian invariant subspaces of Hamiltonian matrices and the related problem of solving algebraic Riccati equations have been extensively studied (see $[12,22,27,35]$ and the references therein), completely satisfactory methods for general Hamiltonian matrices and matrix pencils are still an open problem. Completely satisfactory methods would be numerically backward stable, have complexity $\mathcal{O}\left(n^{3}\right)$ and preserve structure. There are several reasons for this difficulty all of which are well demonstrated in the context of algorithms for Hamiltonian matrices. First of all, an algorithm based upon structure preserving similarity transformations (including $Q R$-like algorithms) would require a triangularlike Hamiltonian Schur form that displays the desired deflating subspaces. A Hamiltonian Schur form under unitary symplectic similarity transformations is presented in [31]. (See (1.1).) Unfortunately, not every Hamiltonian matrix has this kind of Hamiltonian Schur form. For example, the Hamiltonian matrix $\mathcal{J}$ in Definition 1.1 is invariant under arbitrary unitary similarity transformations but is not in the Hamiltonian Schur form described in [31]. (Similar difficulties arise in the skew-Hamiltonian/Hamiltonian pencil case for the Schur-like forms of skew-Hamiltonian/Hamiltonian matrix pencils in [25, 26] and for the other structures given in Definition 1.1 in [24].) A second problem comes from the fact that even when a Hamiltonian Schur form exists, there is no completely satisfactory structure preserving, numerical method to compute it. It has been argued in [2] that, except in special cases [13, 14], $Q R$-like algorithms are impractically expensive because of the lack of a Hamiltonian Hessenberg-like form. For this reason other methods like the multishift-method of [1], the structured implicit product methods of $[6,7,38]$ do not follow the $Q R$-algorithm paradigm. (The implicit product methods $[6,7]$ do come quite close to optimality. We extend the method of [6] to skew-Hamiltonian/Hamiltonian matrix pencils in Section 4.) A third difficulty arises when the Hamiltonian matrix or the skew-Hamiltonian/Hamiltonian matrix pencil has eigenvalues on the imaginary axis. In that case, the desired Lagrangian subspace is, in general, not unique [29]. Furthermore, if finite precision arithmetic or other errors perturb the matrix off the Lie algebra of Hamiltonian matrices, then it is typically the case that the perturbed matrix has no Lagrangian subspace or does not have the expected eigenvalue pairings, see, e.g., $[7,38]$.

We close the introduction by introducing some notation. To simplify notation, the term eigenvalue is used both for eigenvalues of matrices and, in the context of a matrix pencil $\alpha E-\beta A$, for pairs $(\alpha, \beta) \in \mathbb{C} \backslash(0,0)$ for which $\operatorname{det}(\alpha E-\beta A)=0$. These pairs are not unique. If $\beta \neq 0$ then we identify $(\alpha, \beta)$ with $(\alpha / \beta, 1)$ and $\lambda=\alpha / \beta$. Pairs $(\alpha, 0)$ with $\alpha \neq 0$ are called infinite eigenvalues.

By $\Lambda(E, A)$ we denote the set of eigenvalues of $\alpha E-\beta A$ including finite and infinite eigenvalues both counted according to multiplicity. We will denote by $\Lambda_{-}(E, A), \Lambda_{0}(E, A)$ and $\Lambda_{+}(E, A)$ the set of finite eigenvalues of $\alpha A-\beta E$ with negative, zero and positive real parts, respectively. The set of infinite eigenvalues is denoted by $\Lambda_{\infty}(E, A)$. Multiple eigenvalues are repeated in $\Lambda_{-}(E, A)$, $\Lambda_{0}(E, A), \Lambda_{+}(E, A)$ and $\Lambda_{\infty}(E, A)$ according to algebraic multiplicity. The set of all eigenvalues counted according to multiplicity is $\Lambda(E, A):=\Lambda_{-}(E, A) \cup \Lambda_{0}(E, A) \cup \Lambda_{+}(E, A) \cup \Lambda_{\infty}(E, A)$. Similarly, we denote by $\operatorname{Def}_{-}(E, A), \operatorname{Def}_{0}(E, A), \operatorname{Def}_{+}(E, A)$ and $\operatorname{Def}_{\infty}(E, A)$ the right deflating subspaces corresponding to $\Lambda_{-}(E, A), \Lambda_{0}(E, A), \Lambda_{+}(E, A)$ and $\Lambda_{\infty}(E, A)$, respectively. 
Throughout this paper, the imaginary number $\sqrt{-1}$ is denoted by $i$. The inertia of a Hermitian matrix $A$ consists of the triple $\operatorname{In}(A)=(\pi, \omega, \nu)$, where $\pi=\pi(A), \omega=\omega(A)$, and $\nu=\nu(A)$ represent the number of eigenvalues with positive, zero, and negative, real parts, respectively.

By abuse of notation, we identify a subspace and a matrix whose columns span this subspace by the same symbol.

We call a matrix Hamiltonian block triangular if it is Hamiltonian and has the form

$$
\left[\begin{array}{cc}
F & G \\
0 & -F^{H}
\end{array}\right]
$$

If, furthermore, $F$ is triangular then we call the matrix Hamiltonian triangular. The terms skewHamiltonian block triangular and skew-Hamiltonian triangular are defined analogously.

The Hamiltonian (skew-Hamiltonian) Schur form of a Hamiltonian (skew-Hamiltonian) matrix $\mathcal{H}$ is the factorization

$$
\mathcal{H}=\mathcal{U} \mathcal{T U}^{H}
$$

where $\mathcal{U} \in \mathbb{U S}_{2 n}$, and $\mathcal{T}$ is Hamiltonian (skew-Hamiltonian) triangular. As mentioned above, not all Hamiltonian matrices have a Hamiltonian Schur form. Real skew-Hamiltonian matrices always have one [38], but not all complex skew-Hamiltonian matrices do. For Hamiltonian matrices that have no purely imaginary eigenvalues the existence of a Hamiltonian Schur form was proved in [31]. Necessary and sufficient conditions for the existence of the Hamiltonian Schur form in the case of arbitrary spectra were suggested in [23] and a proof based on a structured Hamiltonian Jordan form was recently given in [24].

2. Schur-like forms of Skew-Hamiltonian/Hamiltonian Matrix Pencils. In this section we derive the theoretical background for algorithms to compute eigenvalues and deflating subspaces of skew-Hamiltonian/Hamiltonian matrix pencils. A primary theoretical and computation tool is the $J$-congruence. A $J$-congruence transformation of a $2 n \times 2 n$ pencil $\alpha \mathcal{S}-\beta \mathcal{H}$ by a nonsingular matrix $\mathcal{Y} \in \mathbb{C}^{2 n, 2 n}$ is the conguence transformation $\mathcal{J Y}^{H} \mathcal{J}^{T}(\alpha \mathcal{S}-\beta \mathcal{H}) \mathcal{Y}$ where $\mathcal{J}$ is as in Definition 1.1. The structure of skew-Hamiltonian/Hamiltonian matrix pencils is preserved by $J$-congruence transformations $[25,26]$, i.e., if $\alpha \mathcal{S}-\beta \mathcal{H}$ is a skew-Hamiltonian/Hamiltonian pencil and $\mathcal{Y}$ is nonsingular, then $\mathcal{J Y}^{H} \mathcal{J}^{T}(\alpha \mathcal{S}-\beta \mathcal{H}) \mathcal{Y}$ is also skew-Hamiltonian/Hamiltonian.

The skew-Hamiltonian/Hamiltonian Schur form of a skew-Hamiltonian/Hamiltonian pencil $\alpha \mathcal{S}-\beta \mathcal{H}$ is the factorization

$$
\alpha \mathcal{S}-\beta \mathcal{H}=\mathcal{J} \mathcal{Q J}^{T}\left(\alpha\left[\begin{array}{cc}
S_{11} & S_{12} \\
0 & S_{11}^{H}
\end{array}\right]-\beta\left[\begin{array}{cc}
H_{11} & H_{12} \\
0 & -H_{11}^{H}
\end{array}\right]\right) \mathcal{Q}^{H},
$$

where $\mathcal{Q} \in \mathbb{C}^{2 n, 2 n}$ is unitary, $S_{11} \in \mathbb{C}^{n, n}$ and $H_{11} \in \mathbb{C}^{n, n}$ are upper triangular, $S_{12} \in \mathbb{C}^{n, n}$ is skewHermitian and $H_{12} \in \mathbb{C}^{n, n}$ is Hermitian. Note that the skew-Hamiltonian/Hamiltonian Schur form is a special case of the Schur form a general matrix pencil and that it displays the eigenvalues and a nested system of deflating subspaces. This definition of a skew-Hamiltonian/Hamiltonian Schur form is essentially consistent with the definition of the Hamiltonian Schur form of a Hamiltonian matrix (1.1). If (2.1) holds with $\mathcal{S}=I$, then it is not difficult to show that $\mathcal{Q}$ is a unitary diagonal matrix multiple of a unitary symplectic matrix and that there is a unitary symplectic choice of $\mathcal{Q}$, $\mathcal{Q}^{H}=\mathcal{Q}^{-1}=J \mathcal{Q}^{H} J^{T}$, for which (2.1) holds with $S_{11}=I$ and $S_{12}=0$.

Skew-Hamiltonian/Hamiltonian matrix pencils often have the characteristic that the skewHamiltonian matrix $\mathcal{S}$ is block diagonal $[4,5]$, i.e., $\mathcal{S}=\left[\begin{array}{cc}E & 0 \\ 0 & E^{H}\end{array}\right]$ for some matrix $E \in \mathbb{C}^{n, n}$. In this case (among others), the matrix $\mathcal{S}$ factors in the form

$$
\mathcal{S}=\mathcal{J} \mathcal{Z}^{H} \mathcal{J}^{T} \mathcal{Z}
$$

where $\mathcal{Z}=\operatorname{diag}\left(I, E^{H}\right)$. Such a factorization may also be intrinsic to the problem formulation for non-block diagonal skew-Hamiltonian matrices $\mathcal{S}$; see, e.g., [28]. 
Let $\langle x, y\rangle$ be the indefinite inner product on $\mathbb{C}^{2 n} \times \mathbb{C}^{2 n}$ defined by $\langle x, y\rangle=y^{H} \mathcal{J} x$. If $\mathcal{Z} \in$ $\mathbb{C}^{2 n, 2 n}$, then for all $x, y \in \mathbb{C}^{2 n},\langle(\mathcal{Z} x), y\rangle=\left\langle x,\left(\mathcal{J}^{-T} \mathcal{Z}^{H} \mathcal{J}^{T}\right) y\right\rangle$, i.e., the adjoint of $\mathcal{Z}$ with respect to $\langle$. . . $\rangle$ is $\mathcal{J}^{-T} \mathcal{Z}^{H} \mathcal{J}^{T}$. Because $\mathcal{J}^{-1}=\mathcal{J}^{T}=-\mathcal{J}$, the adjoint may also be expressed as $\mathcal{J Z}^{H} \mathcal{J}^{T}$. From this point of view, (2.2) is a symmetric-like factorization of $\mathcal{S}$ into the product of adjoints $\mathcal{J} \mathcal{Z} \mathcal{J}^{T}$ and $\mathcal{Z}$. By analogy with the factorization of symmetric matrices, we will use the term $\mathcal{J}$-semidefinite to refer to skew-Hamiltonians matrices which have a factorization of the form (2.2). A $\mathcal{J}$-definite skew-Hamiltonian matrix is a skew-Hamiltonian matrix that is both $\mathcal{J}$-semidefinite and non-singular.

The property of $\mathcal{J}$-semidefiniteness arises frequently in applications $[3,4,5]$. We show below that all real skew-Hamiltonian matrices are $\mathcal{J}$-semidefinite. We also show that if a skew-Hamiltonian/Hamiltonian matrix pencil has a skew-Hamiltonian/Hamiltonian Schur form, then the skew-Hamiltonian part is $\mathcal{J}$-semidefinite.

Although $\mathcal{J}$-semidefiniteness is a common property of skew-Hamiltonian matrices it is not universal. The following lemma shows that neither $i \mathcal{J}$ nor any nonsingular, skew-Hamiltonian matrix of the form $i \mathcal{J} L L^{T}$ is $\mathcal{J}$-semidefinite.

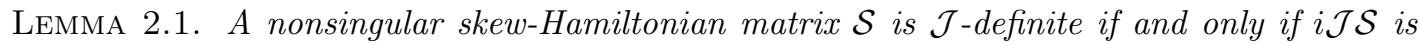
Hermitian with $n$ positive and $n$ negative eigenvalues.

Proof. If $\mathcal{S}$ is $\mathcal{J}$-definite, then $\mathcal{Z}$ in $(2.2)$ is nonsingular and the Hermitian matrix $i \mathcal{J S}$ is congruent to $-i \mathcal{J}^{T}=i \mathcal{J}$. It follows from Sylvester's law of inertia [16, p. 296],[21, p. 188] that $i \mathcal{J S}$ is a Hermitian matrix with $n$ positive eigenvalues and $n$ negative eigenvalues.

Conversely, suppose that $i \mathcal{J S}$ is Hermitian with $n$ positive and $n$ negative eigenvalues. The matrix $i \mathcal{J}^{T}$ also has $n$ positive and $n$ negative eigenvalues, so, by an immediate consequence of Sylvester's law of inertia, there is a nonsingular matrix $\mathcal{Z} \in \mathbb{C}^{2 n, 2 n}$ for which $i \mathcal{J} \mathcal{S}=\mathcal{Z}^{H}\left(i \mathcal{J}^{T}\right) \mathcal{Z}$. It follows that $(2.2)$ holds with this matrix $\mathcal{Z}$.

Lemma 2.1 suggests that $\mathcal{J}$-semidefiniteness might be a characteristic of the inertia of $i \mathcal{J} \mathcal{S}$. The next lemma shows that this is indeed the case.

LEMMA 2.2. A matrix $\mathcal{S} \in \mathbb{S H}_{2 n}$ is $\mathcal{J}$-semidefinite if and only if $i \mathcal{J S}$ satisfies both $\pi(i \mathcal{J S}) \leq$ $n$ and $\nu(i \mathcal{J S}) \leq n$.

Proof. Suppose that $\mathcal{S} \in \mathbb{S H}_{2 n}$ is $\mathcal{J}$-semidefinite. For some $\mathcal{Z}$ satisfying (2.2), define $\mathcal{S}(\epsilon)$ by $\mathcal{S}(\epsilon)=\mathcal{J}(Z+\epsilon I)^{H} \mathcal{J}^{T}(Z+\epsilon I)$. For $\epsilon$ small enough, $\mathcal{Z}+\epsilon I$ is nonsingular, and, by Lemma 2.1, $\pi(i \mathcal{J S}(\epsilon))=n$ and $\nu(i \mathcal{J S}(\epsilon))=n$. Because eigenvalues are continuous functions of matrix elements and $\mathcal{S}=\lim _{\epsilon \rightarrow 0} \mathcal{S}(\epsilon)$, it follows that $\pi(i \mathcal{J S}) \leq n$ and $\nu(i \mathcal{J S}) \leq n$.

For the converse, if $\pi(i \mathcal{J S})=p \leq n$ and $\nu(i \mathcal{J S})=q \leq n$, then, there exists a nonsingular matrix $\mathcal{W}$ for which $i \mathcal{J S}=\mathcal{W}^{H} \mathcal{L} \mathcal{W}$ with signature matrix

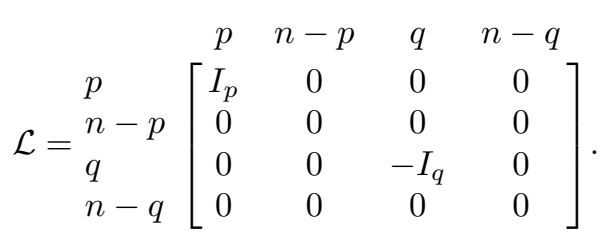

Because $p \leq n$ and $q \leq n, \mathcal{L}$ factors as $\mathcal{L}=\mathcal{L} \operatorname{diag}\left(I_{n},-I_{n}\right) \mathcal{L}$ where $I_{n}$ is the $n \times n$ identity matrix. The matrix $\operatorname{diag}\left(I_{n},-I_{n}\right)$ is the diagonal matrix of eigenvalues of $i \mathcal{J}^{T}$, so $\mathcal{L}=\mathcal{L}\left(\mathcal{U}^{H}\left(i \mathcal{J}^{T}\right) \mathcal{U}\right) \mathcal{L}$, where $\mathcal{U}=(1 / \sqrt{2})\left[\begin{array}{cc}I_{n} & I_{n} \\ i I_{n} & -i I_{n}\end{array}\right]$ is the unitary matrix of eigenvectors of $i \mathcal{J}^{T}$. Hence, (2.2) holds with $\mathcal{Z}=\mathcal{U L W}$.

The following immediate corollary also follows from [15].

Corollary 2.3. Every real skew-Hamiltonian matrix $\mathcal{S}$ is $\mathcal{J}$-semidefinite.

Proof. If $\mathcal{S}$ is real, then $\mathcal{J S}$ is real and skew-symmetric. The eigenvalues of $\mathcal{J S}$ appear in complex conjugate pairs with zero real part. Hence, the eigenvalues of $i \mathcal{J S}$ lie on the real axis in \pm pairs. In particular, $\pi(i \mathcal{J S})=\nu(i \mathcal{J S})$. It follows from the trivial identity $\pi(i \mathcal{J S})+\omega(i \mathcal{J S})+$ $\nu(i \mathcal{J S})=2 n$ that $\pi(i \mathcal{J S}) \leq n$ and $\nu(i \mathcal{J S}) \leq n$

The next lemma and its corollary show that $\mathcal{J}$-semidefiniteness of both $\mathcal{S}$ and $i \mathcal{H}$ are necessary conditions for a skew-Hamiltonian/Hamiltonian matrix pencil $\alpha \mathcal{S}-\beta \mathcal{H}$ to have a skew-Hamiltonian/Hamiltonian Schur. 
LEMMA 2.4. If $\mathcal{S} \in \mathbb{S H}_{2 n}$ and there exists a nonsingular matrix $\mathcal{Y}$ such that

$$
\mathcal{J Y}^{H} \mathcal{J}^{T} \mathcal{S Y}=\left[\begin{array}{cc}
S_{11} & S_{12} \\
0 & S_{11}^{H}
\end{array}\right]
$$

with $S_{11}, S_{12} \in \mathbb{C}^{n, n}$, then $\mathcal{S}$ is $\mathcal{J}$-semidefinite.

Proof. Let $\mathcal{T}$ be the Hermitian matrix

$$
\mathcal{T}=\mathcal{Y}^{H}(i \mathcal{J S}) \mathcal{Y}=\left[\begin{array}{cc}
0 & i S_{11}^{H} \\
-i S_{11} & -i S_{12}
\end{array}\right]
$$

and set $\mathcal{T}(\epsilon)=\mathcal{T}+\epsilon\left[\begin{array}{cc}0 & I_{n} \\ I_{n} & I_{n}\end{array}\right]$. For $\epsilon$ sufficiently small, both $\epsilon I_{n}-i S_{12}$ and $\epsilon I_{n}-i S_{11}$ are nonsingular and $\mathcal{T}(\epsilon)$ is congruent to

$$
\left[\begin{array}{cc}
-\left(\epsilon I_{n}-i S_{11}\right)\left(\epsilon I_{n}-i S_{12}\right)^{-1}\left(\epsilon I_{n}-i S_{11}\right)^{H} & 0 \\
0 & \left(\epsilon I_{n}-i S_{12}\right)
\end{array}\right] .
$$

By Sylvester's law, the inertia of the negative of the $(1,1)$ block is equal to the inertia of the $(2,2)$ block. This implies $\pi(\mathcal{T}(\epsilon))=\nu(\mathcal{T}(\epsilon))=n$. Continuity of eigenvalues as $\epsilon \rightarrow 0$ implies $\pi(\mathcal{T}) \leq n$ and $\nu(\mathcal{T}) \leq n$. The assertion now follows from Lemma 2.2.

Corollary 2.5. If $\mathcal{H} \in \mathbb{H}_{2 n}$ and there exists a nonsingular matrix $\mathcal{Y}$ such that

$$
\mathcal{J Y}^{H} \mathcal{J}^{T} \mathcal{H} \mathcal{Y}=\left[\begin{array}{cc}
H_{11} & H_{12} \\
0 & -H_{11}^{H}
\end{array}\right]
$$

with $H_{11}, H_{12} \in \mathbb{C}^{n, n}$, then $i \mathcal{H}$ is $\mathcal{J}$-semidefinite.

Proof. Apply Lemma 2.4 to the skew-Hamiltonian matrix $i \mathcal{H}$.

It follows from Lemma 2.4 and Corollary 2.5, that if $\alpha \mathcal{S}-\beta \mathcal{H}$ is a skew-Hamiltonian/Hamiltonian matrix pencil that has a skew-Hamiltonian/Hamiltonian Schur form, then $\mathcal{S}$ and $i \mathcal{H}$ are $\mathcal{J}$-semidefinite. As noted above, the factor $\mathcal{Z}$ in $(2.2)$ is often given explicitly as part of the problem statement. It can also be obtained as in the proof of Lemma 2.2 or by a modification of Gaussian elimination [3]. The next theorem shows that if $\mathcal{S}$ is nonsingular, then the skewHamiltonian/Hamiltonian Schur form (if it exists) can be expressed in terms of block triangular factorizations of $\mathcal{Z}$ and $\mathcal{H}$ without explicitly using $\mathcal{S}$. This opens the possibility of designing numerical methods that work directly on $\mathcal{Z}$ and $\mathcal{H}$ and avoid the normal-equations-like numerical instability of forming $\mathcal{S}$ explicitly.

For regular skew-Hamiltonian/Hamiltonian matrix pencils the following theorem gives necessary and sufficient conditions for the existence of a skew-Hamiltonian/Hamiltonian Schur form.

THEOREM 2.6. [25, 26] Let $\alpha \mathcal{S}-\beta \mathcal{H}$ be a regular skew-Hamiltonian/Hamiltonian matrix pencil, with $\nu$ pairwise distinct, finite, nonzero, purely imaginary eigenvalues $i \alpha_{1}, i \alpha_{2}, \ldots, i \alpha_{\nu}$ of algebraic multiplicity $p_{1}, p_{2}, \ldots, p_{\nu}$, and associated right deflating subspaces $\mathcal{Q}_{1}, \mathcal{Q}_{2}, \ldots, \mathcal{Q}_{\nu}$. Let $p_{\infty}$ be the algebraic multiplicity of the eigenvalue infinity and let $\mathcal{Q}_{\infty}$ be its associated deflating subspace. The following are equivalent.

(i) There exists a nonsingular matrix $\mathcal{Y}$, such that

$$
\mathcal{J Y}^{H} \mathcal{J}^{T}(\alpha \mathcal{S}-\beta \mathcal{H}) \mathcal{Y}=\alpha\left[\begin{array}{cc}
S_{11} & S_{12} \\
0 & S_{11}^{H}
\end{array}\right]-\beta\left[\begin{array}{cc}
H_{11} & H_{12} \\
0 & -H_{11}^{H}
\end{array}\right]
$$

where $S_{11}$ and $H_{11}$ are upper triangular while $S_{12}$ is skew-Hermitian and $H_{12}$ is Hermitian.

(ii) There exists a unitary matrix $\mathcal{Q}$ such that $\mathcal{J Q}^{H} \mathcal{J}^{T}(\alpha \mathcal{S}-\beta \mathcal{H}) \mathcal{Q}$ is of the form on the right-hand-side of (2.3).

(iii) For $k=1,2, \ldots, \nu, \mathcal{Q}_{k}^{H} \mathcal{J S} \mathcal{Q}_{k}$ is congruent to a $p_{k} \times p_{k}$ copy of $\mathcal{J}$. (If $\nu=0$, i.e., if $\alpha \mathcal{S}-\beta \mathcal{H}$ has no finite, nonzero, purely imaginary eigenvalue, then this statement holds vacuously.)

Furthermore, if $p_{\infty} \neq 0$ then $\mathcal{Q}_{\infty}^{H} \mathcal{J H} \mathcal{Q}_{\infty}$ is congruent to a $p_{\infty} \times p_{\infty}$ copy of $i \mathcal{J}$. 
Similar results cover real Schur-like forms of real Hamiltonian matrices and skew-Hamiltonian/Hamiltonian matrix pencils [24, 25, 26].

Theorem 2.6 gives necessary and sufficient conditions for existence of a structured triangularlike form for skew-Hamiltonian/Hamiltonian pencils. It also demonstrates that whenever a structured triangular-like form exists, then it also exists under unitary transformations. It is partly because of this fact that there exist structure preserving, numerically stable numerical algorithms like those described here and in [4].

THEOREM 2.7. Let $\alpha \mathcal{S}-\beta \mathcal{H}$ be a skew-Hamiltonian/Hamiltonian matrix pencil with nonsingular, $\mathcal{J}$-semidefinite skew-Hamiltonian part $\mathcal{S}=\mathcal{J Z}^{H} \mathcal{J}^{T} \mathcal{Z}$. If any of the equivalent conditions of Theorem 2.6 holds, then there exists a unitary matrix $\mathcal{Q}$ and a unitary symplectic matrix $\mathcal{U}$ such that

$$
\begin{aligned}
\mathcal{U}^{H} \mathcal{Z} \mathcal{Q} & =\left[\begin{array}{cc}
Z_{11} & Z_{12} \\
0 & Z_{22}
\end{array}\right], \\
\mathcal{J} \mathcal{Q}^{H} \mathcal{J}^{T} \mathcal{H} \mathcal{Q} & =\left[\begin{array}{cc}
H_{11} & H_{12} \\
0 & -H_{11}^{H}
\end{array}\right],
\end{aligned}
$$

where $Z_{11}, Z_{22}^{H}$ and $H_{11}$ are $n \times n$ and upper triangular.

Proof. With $\mathcal{Q}$ as in Theorem 2.6 part (ii) we obtain (2.5) and $\mathcal{J} \mathcal{Q}^{H} \mathcal{J}^{T} \mathcal{S} \mathcal{Q}=\left[\begin{array}{cc}S_{11} & S_{12} \\ 0 & S_{11}^{H}\end{array}\right]$. Partition $\tilde{\mathcal{Z}}=\mathcal{Z} \mathcal{Q}$ as $\tilde{\mathcal{Z}}=\left[Z_{1}, Z_{2}\right]$, where $Z_{1}, Z_{2} \in \mathbb{C}^{2 n, n}$. Using $\mathcal{S}=\mathcal{J} \mathcal{Z}^{H} \mathcal{J}^{T} \mathcal{Z}$, we obtain

$$
\tilde{\mathcal{Z}}^{H} \mathcal{J} \tilde{\mathcal{Z}}=\left[\begin{array}{cc}
0 & S_{11}^{H} \\
-S_{11} & -S_{12}
\end{array}\right]
$$

In particular, $Z_{1}^{H} \mathcal{J} Z_{1}=0$, i.e., the columns of $Z_{1}$ form a basis of a Lagrangian subspace and therefore the columns of $Z_{1}$ form the first $n$ columns of a symplectic matrix. (It is easy to verify from Definition 1.1 that, using the non-negative definite square root, $\left[Z_{1},-\mathcal{J} Z_{1}\left(Z_{1}^{H} Z_{1}\right)^{-1 / 2}\right]$ is symplectic.) It is shown in [11] that $Z_{1}$ has a unitary symplectic $Q R$ factorization

$$
\mathcal{U}^{H} Z_{1}=\left[\begin{array}{c}
Z_{11} \\
0
\end{array}\right]
$$

where $\mathcal{U} \in \mathbb{U S}_{2 n}$ is unitary symplectic and $Z_{11} \in \mathbb{C}^{n, n}$ is upper triangular. Setting

$$
\mathcal{U}^{H} \mathcal{Z} \mathcal{Q}=\mathcal{U}^{H} \tilde{\mathcal{Z}}=\left[\begin{array}{cc}
Z_{11} & Z_{12} \\
0 & Z_{22}
\end{array}\right]
$$

we obtain from (2.6) that $Z_{22}^{H} Z_{11}=S_{11}$. Since $S_{11}$ and $Z_{11}$ are both upper triangular and $Z_{11}$ is nonsingular, we conclude that $Z_{22}^{H}$ is also upper triangular.

Note that the invertibility of $\mathcal{Z}$ is only a sufficient condition for the existence of $\mathcal{U}$ as in (2.4) and (2.5). However, there is no particular pathology associated with $\mathcal{Z}$ being singular. The algorithms described below and in [4] do not require $\mathcal{Z}$ to be nonsingular.

If both $\mathcal{S}$ and $\mathcal{H}$ are nonsingular, then the following stronger form of Theorem 2.7 holds.

COROLlary 2.8. Let $\alpha \mathcal{S}-\beta \mathcal{H}$ be a skew-Hamiltonian/Hamiltonian matrix pencil with nonsingular $\mathcal{J}$-semidefinite skew-Hamiltonian part $\mathcal{S}=\mathcal{J Z}^{H} \mathcal{J}^{T} \mathcal{Z}$ and nonsingular $\mathcal{J}$-semidefinite Hamiltonian part $i \mathcal{H}=\mathcal{J} \mathcal{W}^{H} \mathcal{J}^{T} \mathcal{W}$. If any of the equivalent conditions of Theorem 2.6 holds, then there exist a unitary matrix $\mathcal{Q}$ and unitary symplectic matrices $\mathcal{U}$ and $\mathcal{V}$ such that

$$
\mathcal{U}^{H} \mathcal{Z} \mathcal{Q}=\left[\begin{array}{cc}
Z_{11} & Z_{12} \\
0 & Z_{22}
\end{array}\right], \quad \mathcal{V}^{H} \mathcal{W} \mathcal{Q}=\left[\begin{array}{cc}
W_{11} & W_{12} \\
0 & W_{22}
\end{array}\right]
$$

where $Z_{11}, Z_{22}^{H}$ and $W_{11}, W_{22}^{H}$ are $n \times n$ and upper triangular.

Proof. Similar to the proof of Theorem 2.7 .

In the following we derive the theoretical background for algorithms to compute eigenvalues and deflating subspaces of skew-Hamiltonian/Hamiltonian matrix pencils. 
We will obtain the structured Schur form of a complex skew-Hamiltonian/Hamiltonian matrix pencil from the structured Schur form of a real skew-Hamiltonian/skew-Hamiltonian matrix pencil of double dimension. The following theorem establishes that, in contrast to the complex skew-Hamiltonian/Hamiltonian case, every real, regular skew-Hamiltonian/skew-Hamiltonian pencil admits a structured real Schur form.

THEOREM 2.9. If $\alpha \mathcal{S}-\beta \mathcal{N}$ is a real, regular skew-Hamiltonian/skew-Hamiltonian matrix pencil with $\mathcal{S}=\mathcal{J Z}^{T} \mathcal{J}^{T} \mathcal{Z}$, then there exist a real orthogonal matrix $\mathcal{Q} \in \mathbb{R}^{2 n, 2 n}$ and a real orthogonal symplectic matrix $\mathcal{U} \in \mathbb{R}^{2 n, 2 n}$ such that

$$
\begin{aligned}
\mathcal{U}^{T} \mathcal{Z} \mathcal{Q} & =\left[\begin{array}{cc}
Z_{11} & Z_{12} \\
0 & Z_{22}
\end{array}\right], \\
\mathcal{J} \mathcal{Q}^{T} \mathcal{J}^{T} \mathcal{N} \mathcal{Q} & =\left[\begin{array}{cc}
N_{11} & N_{12} \\
0 & N_{11}^{T}
\end{array}\right],
\end{aligned}
$$

where $Z_{11}$ and $Z_{22}^{T}$ are upper triangular, $N_{11}$ is quasi upper triangular and $N_{12}$ is skew-symmetric.

Moreover,

$$
\mathcal{J} \mathcal{Q}^{T} \mathcal{J}^{T}(\alpha \mathcal{S}-\beta \mathcal{N}) \mathcal{Q}=\alpha\left[\begin{array}{cc}
Z_{22}^{T} Z_{11} & Z_{22}^{T} Z_{12}-Z_{12}^{T} Z_{22} \\
0 & Z_{11}^{T} Z_{22}
\end{array}\right]-\beta\left[\begin{array}{cc}
N_{11} & N_{12} \\
0 & N_{11}^{T}
\end{array}\right]
$$

is a $\mathcal{J}$-congruent skew-Hamiltonian/skew-Hamiltonian matrix pencil.

Proof. A constructive proof for the existence of $\mathcal{Q}$ and $\mathcal{U}$ satisfying (2.7) and (2.8) is Algorithm 3 in [4]. To show (2.9), recall that $\mathcal{U}$ is orthogonal symplectic and therefore commutes with $\mathcal{J}$. Hence,

$$
\begin{aligned}
\mathcal{J} \mathcal{Q}^{T} \mathcal{J}^{T} \mathcal{S} \mathcal{Q} & =\mathcal{J} \mathcal{Q}^{T} \mathcal{J}^{T}\left(\mathcal{J} \mathcal{Z}^{T} \mathcal{J}^{T} \mathcal{Z}\right) \mathcal{Q} \\
& =\mathcal{J} \mathcal{Q}^{T} \mathcal{J}^{T}\left(\mathcal{J} \mathcal{Z}^{T} \mathcal{J}^{T} \mathcal{U}\right)\left(\mathcal{U}^{T} \mathcal{Z} \mathcal{Q}\right) \\
& =\mathcal{J}\left(\mathcal{U}^{T} \mathcal{Z} \mathcal{Q}\right)^{T} \mathcal{J}^{T}\left(\mathcal{U}^{T} \mathcal{Z} \mathcal{Q}\right)
\end{aligned}
$$

Equation (2.9) now follows from the block triangular form of (2.7).

Note that this theorem does not easily extend to complex skew-Hamiltonian/skew-Hamiltonian matrix pencils.

A method for computing the structured Schur form (2.9) for real matrices was proposed in [32], but if $\mathcal{S}$ is given in factored form, then Algorithm 3 in [4] is more robust in finite precision arithmetic, because it avoids forming $\mathcal{S}$ explicitly.

Neither the method in [32] nor Algorithm 3 in [4] applies to complex skew-Hamiltonian/Hamiltonian matrix pencils because those algorithms depend on the fact that real diagonal skewsymmetric matrices are identically zero. This property is also crucial for the structured Schur form algorithms in [6,38].

Algorithm 1 given below computes the eigenvalues of a complex skew-Hamiltonian/Hamiltonian matrix pencil $\alpha \mathcal{S}-\beta \mathcal{H}$ using an unusual embedding of $\mathbb{C}$ into $\mathbb{R}^{2}$ that was recently proposed in [8]. Let $\alpha \mathcal{S}-\beta \mathcal{H}$ be a complex skew-Hamiltonian/Hamiltonian matrix pencil with $\mathcal{J}$-semidefinite skew-Hamiltonian part $\mathcal{S}=\mathcal{J Z}^{H} \mathcal{J}^{T} \mathcal{Z}$. Split the skew-Hamiltonian matrix $\mathcal{N}=i \mathcal{H} \in \mathbb{S H}_{2 n}$ as $i \mathcal{H}=\mathcal{N}=\mathcal{N}_{1}+i \mathcal{N}_{2}$, where $\mathcal{N}_{1}$ is real skew-Hamiltonian and $\mathcal{N}_{2}$ is real Hamiltonian, i.e.,

$$
\begin{aligned}
& \mathcal{N}_{1}=\left[\begin{array}{ll}
F_{1} & G_{1} \\
H_{1} & F_{1}^{T}
\end{array}\right], \quad G_{1}=-G_{1}^{T}, \quad H_{1}=-H_{1}^{T}, \\
& \mathcal{N}_{2}=\left[\begin{array}{cc}
F_{2} & G_{2} \\
H_{2} & -F_{2}^{T}
\end{array}\right], \quad G_{2}=G_{2}^{T}, \quad H_{2}=H_{2}^{T},
\end{aligned}
$$


and $F_{j}, G_{j}, H_{j} \in \mathbb{R}^{n \times n}$ for $j=1,2$. Setting

$$
\begin{aligned}
\mathcal{Y}_{c} & =\frac{\sqrt{2}}{2}\left[\begin{array}{cc}
I_{2 n} & i I_{2 n} \\
I_{2 n} & -i I_{2 n}
\end{array}\right], \\
\mathcal{P} & =\left[\begin{array}{cccc}
I_{n} & 0 & 0 & 0 \\
0 & 0 & I_{n} & 0 \\
0 & I_{n} & 0 & 0 \\
0 & 0 & 0 & I_{n}
\end{array}\right], \\
\mathcal{X}_{c} & =\mathcal{Y}_{c} \mathcal{P},
\end{aligned}
$$

and using the embedding $\mathcal{B}_{\mathcal{N}}=\operatorname{diag}(\mathcal{N}, \overline{\mathcal{N}})$ we obtain that

$$
\mathcal{B}_{\mathcal{N}}^{c}:=\mathcal{X}_{c}^{H} \mathcal{B}_{\mathcal{N}} \mathcal{X}_{c}=\left[\begin{array}{cc|cc}
F_{1} & -F_{2} & G_{1} & -G_{2} \\
F_{2} & F_{1} & G_{2} & G_{1} \\
\hline H_{1} & -H_{2} & F_{1}^{T} & F_{2}^{T} \\
H_{2} & H_{1} & -F_{2}^{T} & F_{1}^{T}
\end{array}\right]
$$

is a real skew-Hamiltonian matrix in $\mathbb{S H}_{4 n}$. Similarly, set

$$
\begin{aligned}
\mathcal{B}_{\mathcal{Z}} & :=\left[\begin{array}{cc}
\mathcal{Z} & 0 \\
0 & \overline{\mathcal{Z}}
\end{array}\right], \\
\mathcal{B}_{\mathcal{T}} & :=\left[\begin{array}{cc}
\mathcal{J Z}^{H} \mathcal{J}^{T} & \frac{0}{\mathcal{J} \mathcal{Z}^{H} \mathcal{J}^{T}}
\end{array}\right] \\
0 & \\
\mathcal{B}_{\mathcal{S}} & :=\left[\begin{array}{cc}
\mathcal{S} & 0 \\
0 & \overline{\mathcal{S}}
\end{array}\right]=\mathcal{B}_{\mathcal{T}} \mathcal{B}_{\mathcal{Z}} .
\end{aligned}
$$

Hence,

$$
\alpha \mathcal{B}_{\mathcal{S}}-\beta \mathcal{B}_{\mathcal{N}}=\left[\begin{array}{cc}
\alpha \mathcal{S}-\beta \mathcal{N} & 0 \\
0 & \alpha \overline{\mathcal{S}}-\beta \overline{\mathcal{N}}
\end{array}\right]
$$

One can easily verify that

$$
\begin{aligned}
\mathcal{B}_{\mathcal{Z}}^{c} & :=\mathcal{X}_{c}^{H} \mathcal{B}_{\mathcal{Z}} \mathcal{X}_{c} \\
\mathcal{B}_{\mathcal{T}}^{c} & :=\mathcal{X}_{c}^{H} \mathcal{B}_{\mathcal{T}} \mathcal{X}_{c}=\mathcal{J}\left(\mathcal{B}_{\mathcal{Z}}^{c}\right)^{T} \mathcal{J}^{T} \\
\mathcal{B}_{\mathcal{S}}^{c} & :=\mathcal{X}_{c}^{H} \mathcal{B}_{\mathcal{S}} \mathcal{X}_{c}=\mathcal{J}\left(\mathcal{B}_{\mathcal{Z}}^{c}\right)^{T} \mathcal{J}^{T} \mathcal{B}_{\mathcal{Z}}^{c}
\end{aligned}
$$

are all real. Therefore,

$$
\begin{aligned}
\alpha \mathcal{B}_{\mathcal{S}}^{c}-\beta \mathcal{B}_{\mathcal{N}}^{c} & =\mathcal{X}_{c}^{H}\left(\alpha \mathcal{B}_{\mathcal{S}}-\beta \mathcal{B}_{\mathcal{N}}\right) \mathcal{X}_{c} \\
& =\mathcal{X}_{c}^{H}\left[\begin{array}{cc}
\alpha \mathcal{S}-\beta \mathcal{N} & 0 \\
0 & \alpha \overline{\mathcal{S}}-\beta \overline{\mathcal{N}}
\end{array}\right] \mathcal{X}_{c}
\end{aligned}
$$

is a real $4 n \times 4 n$ skew-Hamiltonian/skew-Hamiltonian matrix pencil. For this matrix pencil we can employ Algorithm 3 in [4] to compute the structured factorization (2.8), i.e., we can determine an orthogonal symplectic matrix $\mathcal{U}$ and an orthogonal matrix $\mathcal{Q}$ such that

$$
\begin{aligned}
& \tilde{\mathcal{B}}_{\mathcal{Z}}^{c}:=\mathcal{U}^{T} \mathcal{B}_{\mathcal{Z}}^{c} \mathcal{Q}=\left[\begin{array}{cc}
\mathcal{Z}_{11} & \mathcal{Z}_{12} \\
0 & \mathcal{Z}_{22}
\end{array}\right] \\
& \tilde{\mathcal{B}}_{\mathcal{N}}^{c}:=\mathcal{J} \mathcal{Q}^{T} \mathcal{J}^{T} \mathcal{B}_{\mathcal{N}}^{c} \mathcal{Q}=\left[\begin{array}{cc}
\mathcal{N}_{11} & \mathcal{N}_{12} \\
0 & \mathcal{N}_{11}^{T}
\end{array}\right]
\end{aligned}
$$

Thus, if $\tilde{\mathcal{B}}_{\mathcal{S}}^{c}:=\mathcal{J}\left(\tilde{\mathcal{B}}_{\mathcal{Z}}^{c}\right)^{T} \mathcal{J}^{T} \tilde{\mathcal{B}}_{\mathcal{Z}}^{c}$, then

$$
\alpha \tilde{\mathcal{B}}_{\mathcal{S}}^{c}-\beta \tilde{\mathcal{B}}_{\mathcal{N}}^{c}=\alpha\left(\mathcal{J} \mathcal{Q}^{T} \mathcal{J}^{T} \mathcal{B}_{\mathcal{S}}^{c} \mathcal{Q}\right)-\beta\left(\mathcal{J} \mathcal{Q}^{T} \mathcal{J}^{T} \mathcal{B}_{\mathcal{N}}^{c} \mathcal{Q}\right)
$$


is a $\mathcal{J}$-congruent skew-Hamiltonian/skew-Hamiltonian matrix pencil in Schur form. By (2.18) and the fact that the finite eigenvalues of $\alpha \mathcal{S}-\beta \mathcal{N}$ are symmetric with respect to the real axis, we observe that the spectrum of the extended matrix pencil $\alpha \mathcal{B}_{\mathcal{S}}^{c}-\beta \mathcal{B}_{\mathcal{N}}^{c}$ consists of two copies of the spectrum of $\alpha \mathcal{S}-\beta \mathcal{N}$. Consequently,

$$
\Lambda(\mathcal{S}, \mathcal{H})=\Lambda(\mathcal{S},-i \mathcal{N})=\Lambda\left(\mathcal{Z}_{22}^{T} \mathcal{Z}_{11},-i \mathcal{N}_{11}\right)
$$

In this way, Algorithm 1 below computes the eigenvalues of the complex skew-Hamiltonian/Hamiltonian matrix pencil $\alpha \mathcal{S}-\beta \mathcal{H}=\alpha \mathcal{S}+i \beta \mathcal{N}$.

From this we can also derive the skew-Hamiltonian/Hamiltonian Schur form of $\alpha \mathcal{B}_{\mathcal{S}}-\beta \mathcal{B}_{\mathcal{H}}$ where

$$
\mathcal{B}_{\mathcal{H}}=-i \mathcal{B}_{\mathcal{N}}=\left[\begin{array}{cc}
\mathcal{H} & 0 \\
0 & -\overline{\mathcal{H}}
\end{array}\right]
$$

and $\mathcal{B}_{\mathcal{S}}$ is as in (2.17). The spectrum of the extended matrix pencil $\alpha \mathcal{B}_{\mathcal{S}}-\beta \mathcal{B}_{\mathcal{H}}$ consists of two copies of the spectrum of $\alpha \mathcal{S}-\beta \mathcal{H}[6]$. If

$$
\mathcal{B}_{\mathcal{H}}^{c}=-i \mathcal{B}_{\mathcal{N}}^{c}=\mathcal{X}_{c}^{H} \mathcal{B}_{\mathcal{H}} \mathcal{X}_{c}
$$

then it follows from (2.19) and (2.20) that

$$
\begin{aligned}
\tilde{\mathcal{B}}_{\mathcal{Z}}^{c} & :=\mathcal{U}^{T} \mathcal{B}_{\mathcal{Z}}^{c} \mathcal{Q}=\left[\begin{array}{cc}
\mathcal{Z}_{11} & \mathcal{Z}_{12} \\
0 & \mathcal{Z}_{22}
\end{array}\right], \\
\tilde{\mathcal{B}}_{\mathcal{H}}^{c} & :=\mathcal{J} \mathcal{Q}^{T} \mathcal{J}^{T} \mathcal{B}_{\mathcal{H}}^{c} \mathcal{Q}=\left[\begin{array}{cc}
-i \mathcal{N}_{11} & -i \mathcal{N}_{12} \\
0 & -\left(-i \mathcal{N}_{11}\right)^{H}
\end{array}\right],
\end{aligned}
$$

and the matrix pencil $\alpha \tilde{\mathcal{B}}_{\mathcal{S}}^{c}-\beta \tilde{\mathcal{B}}_{\mathcal{H}}^{c}:=\alpha \mathcal{J}\left(\tilde{\mathcal{B}}_{\mathcal{Z}}^{c}\right)^{H} \mathcal{J}^{T} \tilde{\mathcal{B}}_{\mathcal{Z}}^{c}-\beta \tilde{\mathcal{B}}_{\mathcal{H}}^{c}$ is in skew-Hamiltonian/Hamiltonian Schur form. We have thus obtained the structured Schur form of the extended complex skew-Hamiltonian/Hamiltonian matrix pencil $\alpha \mathcal{B}_{\mathcal{S}}^{c}-\beta \mathcal{B}_{\mathcal{H}}^{c}$. Moreover,

$$
\alpha \tilde{\mathcal{B}}_{\mathcal{S}}^{c}-\beta \tilde{\mathcal{B}}_{\mathcal{H}}^{c}=\mathcal{J} \mathcal{Q}^{H} \mathcal{J}^{T}\left(\alpha \mathcal{B}_{\mathcal{S}}^{c}-\beta \mathcal{B}_{\mathcal{H}}^{c}\right) \mathcal{Q}=\left(\mathcal{X}_{c} \mathcal{J} \mathcal{Q} \mathcal{J}^{T}\right)^{H}\left(\alpha \mathcal{B}_{\mathcal{S}}-\beta \mathcal{B}_{\mathcal{H}}\right) \mathcal{X}_{c} \mathcal{Q}
$$

is in skew-Hamiltonian/Hamiltonian Schur form.

We have seen so far that we can compute structured Schur forms and thus are able to compute the eigenvalues of the structured matrix pencils under consideration using the embedding technique into a structured matrix pencil of double size.

3. Deflating Subspaces of Skew-Hamiltonian/Hamiltonian Matrix Pencils. For the solution of problems involving skew-Hamiltonian/Hamiltonian matrix pencils as described in the introduction it is usually necessary to compute $n$-dimensional deflating subspaces associated with eigenvalues in the closed left half plane. To get the desired subspaces we generalize the techniques developed in [6]. For this we need a structure preserving method to reorder the eigenvalues along the diagonal of the structured Schur form so that all eigenvalues with negative real part appear in the $(1,1)$ block and eigenvalues with positive real part appear in the $(2,2)$ block. Such a reordering method is described in Appendix B of [4].

The following theorem uses this eigenvalue ordering to determine the desired deflating subspaces of the matrix pencil $\alpha \mathcal{S}-\beta \mathcal{H}$ from the structured Schur form (2.25).

TheOREm 3.1. Let $\alpha \mathcal{S}-\beta \mathcal{H} \in \mathbb{C}^{2 n, 2 n}$ be a skew-Hamiltonian/Hamiltonian matrix pencil with $\mathcal{J}$-semidefinite skew-Hamiltonian matrix $\mathcal{S}=\mathcal{J Z}^{H} \mathcal{J}^{T} \mathcal{Z}$. Consider the extended matrices

$$
\begin{aligned}
\mathcal{B}_{\mathcal{Z}} & =\operatorname{diag}(\mathcal{Z}, \overline{\mathcal{Z}}), \\
\mathcal{B}_{\mathcal{T}} & =\operatorname{diag}\left(\mathcal{J} \mathcal{Z}^{H} \mathcal{J}^{T}, \overline{\mathcal{J} \mathcal{Z}^{H} \mathcal{J}^{T}}\right), \\
\mathcal{B}_{\mathcal{S}} & =\mathcal{B}_{\mathcal{T}} \mathcal{B}_{\mathcal{Z}}=\operatorname{diag}(\mathcal{S}, \overline{\mathcal{S}}), \\
\mathcal{B}_{\mathcal{H}} & =\operatorname{diag}(\mathcal{H},-\overline{\mathcal{H}}) .
\end{aligned}
$$


Let $\mathcal{U}, \mathcal{V}, \mathcal{W}$ be unitary matrices such that

$$
\begin{aligned}
& \mathcal{U}^{H} \mathcal{B}_{\mathcal{Z}} \mathcal{V}=\left[\begin{array}{cc}
\mathcal{Z}_{11} & \mathcal{Z}_{12} \\
0 & \mathcal{Z}_{22}
\end{array}\right]=: \mathcal{R}_{\mathcal{Z}}, \\
& \mathcal{W}^{H} \mathcal{B}_{\mathcal{T}} \mathcal{U}=\left[\begin{array}{cc}
\mathcal{T}_{11} & \mathcal{T}_{12} \\
0 & \mathcal{T}_{22}
\end{array}\right]=: \mathcal{R}_{\mathcal{T}}, \\
& \mathcal{W}^{H} \mathcal{B}_{\mathcal{H}} \mathcal{V}=\left[\begin{array}{cc}
\mathcal{H}_{11} & \mathcal{H}_{12} \\
0 & \mathcal{H}_{22}
\end{array}\right]=: \mathcal{R}_{\mathcal{H}},
\end{aligned}
$$

where $\Lambda_{-}\left(\mathcal{B}_{\mathcal{S}}, \mathcal{B}_{\mathcal{H}}\right) \subset \Lambda\left(\mathcal{T}_{11} \mathcal{Z}_{11}, \mathcal{H}_{11}\right)$ and $\Lambda\left(\mathcal{T}_{11} \mathcal{Z}_{11}, \mathcal{H}_{11}\right) \cap \Lambda_{+}\left(\mathcal{B}_{\mathcal{S}}, \mathcal{B}_{\mathcal{H}}\right)=\emptyset$. Here $\mathcal{Z}_{11}, \mathcal{T}_{11}, \mathcal{H}_{11} \in$ $\mathbb{C}^{m, m}$. Suppose $\Lambda_{-}(\mathcal{S}, \mathcal{H})$ contains p eigenvalues. If $\left[\begin{array}{l}V_{1} \\ V_{2}\end{array}\right] \in \mathbb{C}^{4 n, m}$ are the first $m$ columns of $\mathcal{V}$, $2 p \leq m \leq 2 n-2 p$, then there are subspaces $\mathbb{L}_{1}$ and $\mathbb{L}_{2}$ such that

$$
\begin{array}{lll}
\text { range } V_{1}=\operatorname{Def}_{-}(\mathcal{S}, \mathcal{H})+\mathbb{L}_{1}, & \mathbb{L}_{1} \subseteq \operatorname{Def}_{0}(\mathcal{S}, \mathcal{H})+\operatorname{Def}_{\infty}(\mathcal{S}, \mathcal{H}), \\
\text { range } \overline{V_{2}}=\operatorname{Def}_{+}(\mathcal{S}, \mathcal{H})+\mathbb{L}_{2}, & \mathbb{L}_{2} \subseteq \operatorname{Def}_{0}(\mathcal{S}, \mathcal{H})+\operatorname{Def}_{\infty}(\mathcal{S}, \mathcal{H})
\end{array}
$$

If $\Lambda\left(\mathcal{T}_{11} \mathcal{Z}_{11}, \mathcal{H}_{11}\right)=\Lambda_{-}\left(\mathcal{B}_{\mathcal{S}}, \mathcal{B}_{\mathcal{H}}\right)$, and $\left[\begin{array}{c}U_{1} \\ U_{2}\end{array}\right],\left[\begin{array}{c}W_{1} \\ W_{2}\end{array}\right]$ are the first $m$ columns of $\mathcal{U}, \mathcal{W}$, respectively, then there exist unitary matrices $Q_{U}, Q_{V}, Q_{W}$ such that

$$
\begin{aligned}
U_{1} & =\left[P_{U}^{-}, 0\right] Q_{U}, & U_{2} & =\left[0, P_{U}^{+}\right] Q_{U}, \\
V_{1} & =\left[P_{V}^{-}, 0\right] Q_{V}, & V_{2} & =\left[0, P_{V}^{+}\right] Q_{V}, \\
W_{1} & =\left[P_{W}^{-}, 0\right] Q_{W}, & W_{2} & =\left[0, P_{W}^{+}\right] Q_{W}
\end{aligned}
$$

and the columns of $P_{V}^{-}$and $\overline{P_{V}^{+}}$form orthogonal bases of $\operatorname{Def}_{-}(\mathcal{S}, \mathcal{H})$ and $\operatorname{Def}_{+}(\mathcal{S}, \mathcal{H})$, respectively. Moreover, the matrices $P_{U}^{-}, P_{U}^{+}, P_{W}^{-}$and $P_{W}^{+}$have orthonormal columns and the following relations are satisfied

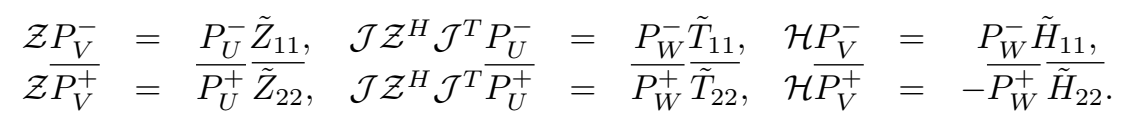

Here, $\tilde{Z}_{k k}, \tilde{T}_{k k}$ and $\tilde{H}_{k k}, k=1,2$, satisfy $\Lambda\left(\tilde{T}_{11} \tilde{Z}_{11}, \tilde{H}_{11}\right)=\Lambda\left(\tilde{T}_{22} \tilde{Z}_{22}, \tilde{H}_{22}\right)=\Lambda_{-}(\mathcal{S}, \mathcal{H})$.

Proof. The factorizations in (3.1) imply that $\mathcal{B}_{\mathcal{S}} \mathcal{V}=\mathcal{W} \mathcal{R}_{\mathcal{T}} \mathcal{R}_{\mathcal{Z}}$ and $\mathcal{B}_{\mathcal{H}} \mathcal{V}=\mathcal{W} \mathcal{R}_{\mathcal{H}}$. Comparing the first $m$ columns and making use of the block forms we have

$$
\begin{aligned}
& \mathcal{S} V_{1}=W_{1}\left(\mathcal{T}_{11} \mathcal{Z}_{11}\right), \\
& \mathcal{S} \overline{V_{2}}=\bar{H} V_{1}=W_{2}\left(\overline{\mathcal{T}}_{11} \mathcal{Z}_{11}\right),
\end{aligned}
$$

Clearly, range $V_{1}$ and range $\overline{V_{2}}$ are both deflating subspaces of $\alpha \mathcal{S}-\beta \mathcal{H}$. Since

$$
\Lambda_{-}(\mathcal{S}, \mathcal{H}) \subseteq \Lambda_{-}\left(\mathcal{B}_{\mathcal{S}}, \mathcal{B}_{\mathcal{H}}\right) \subseteq \Lambda\left(\mathcal{T}_{11} \mathcal{Z}_{11}, \mathcal{H}_{11}\right)
$$

and $\Lambda\left(\mathcal{T}_{11} \mathcal{Z}_{11}, \mathcal{H}_{11}\right)$ contains no eigenvalue with positive real part, we get

$$
\begin{array}{ll}
\text { range } V_{1} \subseteq \operatorname{Def}_{-}(\mathcal{S}, \mathcal{H})+\mathbb{L}_{1}, & \mathbb{L}_{1} \subseteq \operatorname{Def}_{0}(\mathcal{S}, \mathcal{H})+\operatorname{Def}_{\infty}(\mathcal{S}, \mathcal{H}), \\
\text { range } \overline{V_{2}} \subseteq \operatorname{Def}_{+}(\mathcal{S}, \mathcal{H})+\mathbb{L}_{2}, & \mathbb{L}_{2} \subseteq \operatorname{Def}_{0}(\mathcal{S}, \mathcal{H})+\operatorname{Def}_{\infty}(\mathcal{S}, \mathcal{H})
\end{array}
$$

We still need to show that

$$
\operatorname{Def}_{-}(\mathcal{S}, \mathcal{H}) \subseteq \text { range } V_{1}, \quad \operatorname{Def}_{+}(\mathcal{S}, \mathcal{H}) \subseteq \text { range } \overline{V_{2}}
$$

Let $\tilde{V}_{1}$ and $\tilde{V}_{2}$ be full rank matrices whose columns form bases of Def- $(\mathcal{S}, \mathcal{H})$ and $\operatorname{Def}_{+}(\mathcal{S}, \mathcal{H})$, respectively. It is easy to show that the columns of $\left[\begin{array}{cc}\tilde{V}_{1} & 0 \\ 0 & \tilde{V}_{2}\end{array}\right]$ span Def $-\left(\mathcal{B}_{\mathcal{S}}, \mathcal{B}_{\mathcal{H}}\right)$. This implies that

$$
\operatorname{range}\left[\begin{array}{cc}
\tilde{V}_{1} & 0 \\
0 & \tilde{V}_{2}
\end{array}\right] \subseteq \text { range }\left[\begin{array}{c}
V_{1} \\
V_{2}
\end{array}\right]
$$


Therefore,

$$
\operatorname{range}\left[\begin{array}{c}
\tilde{V}_{1} \\
0
\end{array}\right], \text { range }\left[\frac{0}{\tilde{V}_{2}}\right] \subseteq \text { range }\left[\begin{array}{c}
V_{1} \\
V_{2}
\end{array}\right]
$$

and from this we obtain (3.5) and hence (3.2).

If $\Lambda\left(\mathcal{T}_{11} \mathcal{Z}_{11}, \mathcal{H}_{11}\right)=\Lambda_{-}\left(\mathcal{B}_{\mathcal{S}}, \mathcal{B}_{\mathcal{H}}\right)$, where $p$ is the number of eigenvalues in $\Lambda_{-}(\mathcal{S}, \mathcal{H})$, then from (3.2) we have $m=2 p$ and

$$
\text { range } V_{1}=\operatorname{Def}_{-}(\mathcal{S}, \mathcal{H}), \quad \text { range } \overline{V_{2}}=\operatorname{Def}_{+}(\mathcal{S}, \mathcal{H})
$$

Hence, rank $V_{1}=\operatorname{rank} V_{2}=p$ and furthermore $\mathcal{T}_{11}, \mathcal{Z}_{11}$ and $\mathcal{H}_{11}$ must be nonsingular. Using (3.4) we get

$$
\begin{aligned}
& \mathcal{H} V_{1}=\mathcal{S} V_{1}\left(\left(\mathcal{T}_{11} \mathcal{Z}_{11}\right)^{-1} \mathcal{H}_{11}\right) \\
& \mathcal{H} \overline{V_{2}}=-\mathcal{S} \overline{V_{2}}\left(\overline{\left(\mathcal{T}_{11} \mathcal{Z}_{11}\right)^{-1} \mathcal{H}_{11}}\right) .
\end{aligned}
$$

Let $V_{1}=\left[P_{V}^{-}, 0\right] Q_{V}$ be an $R Q$ (triangular-orthogonal) decomposition [17] with $P_{V}^{-}$of full column rank. Since rank $V_{1}=p$ we have rank $P_{V}^{-}=p$. Partition $V_{2} Q_{V}^{H}=\left[P_{V}, P_{V}^{+}\right]$conforming to $V_{1} Q_{V}^{H}$. Since the columns of $\left[\begin{array}{l}V_{1} \\ V_{2}\end{array}\right]$ are orthonormal, we obtain $\left(P_{V}^{+}\right)^{H} P_{V}^{+}=I_{p}$ and hence $\operatorname{rank} P_{V}^{+}=p$. Furthermore, since rank $V_{2}=p$ we have

$$
\text { range } P_{V} \subseteq \text { range } P_{V}^{+}=\text {range } V_{2},
$$

and using orthonormality, we obtain $P_{V}=0$. Therefore, the columns of $P_{V}^{-}$and $\overline{P_{V}^{+}}$form orthogonal bases of $\operatorname{Def}_{-}(\mathcal{S}, \mathcal{H})$ and $\operatorname{Def}_{+}(\mathcal{S}, \mathcal{H})$, respectively.

From (3.1) we have

$$
\mathcal{Z} V_{1}=U_{1} \mathcal{Z}_{11}, \quad \mathcal{J} \mathcal{Z}^{H} \mathcal{J}^{T} U_{1}=W_{1} \mathcal{T}_{11}, \quad \mathcal{H} V_{1}=W_{1} \mathcal{H}_{11}
$$

and

$$
\mathcal{Z} \overline{V_{2}}=\overline{U_{2}} \overline{\mathcal{Z}_{11}}, \quad \mathcal{J} \mathcal{Z}^{H} \mathcal{J}^{T} \overline{U_{2}}=\overline{W_{2}} \overline{\mathcal{T}_{11}}, \quad \mathcal{H} \overline{V_{2}}=-\overline{W_{2}} \overline{\mathcal{H}_{11}}
$$

Let $U_{1}=\left[P_{U}^{-}, 0\right] Q_{U}$ and $W_{1}=\left[P_{W}^{-}, 0\right] Q_{W}$ be $R Q$ (triangular-orthogonal) decompositions, with $P_{U}^{-}, P_{W}^{-}$of full column rank. Using $V_{1}=\left[P_{V}^{-}, 0\right] Q_{V}$ and the fact that $\mathcal{Z} P_{V}^{-}, \mathcal{S} P_{V}^{-}$and $\mathcal{H} P_{V}^{-}$are of full rank (otherwise there would be a zero or infinite eigenvalue associated with the deflating subspace range $P_{V}^{-}$), from the first and third identity in (3.6) we obtain

$$
\operatorname{rank} P_{U}^{-}=\operatorname{rank} P_{W}^{-}=\operatorname{rank} P_{V}^{-}=p
$$

Moreover, setting

$$
\tilde{Z}=Q_{U} \mathcal{Z}_{11} Q_{V}^{H}, \quad \tilde{T}=Q_{W} \mathcal{T}_{11} Q_{U}^{H}, \quad \tilde{H}=Q_{W} \mathcal{H}_{11} Q_{V}^{H}
$$

we obtain

$$
\tilde{Z}=\left[\begin{array}{cc}
\tilde{Z}_{11} & 0 \\
\tilde{Z}_{21} & \tilde{Z}_{22}
\end{array}\right], \quad \tilde{T}=\left[\begin{array}{cc}
\tilde{T}_{11} & 0 \\
\tilde{T}_{21} & \tilde{T}_{22}
\end{array}\right], \quad \tilde{H}=\left[\begin{array}{cc}
\tilde{H}_{11} & 0 \\
\tilde{H}_{21} & \tilde{H}_{22}
\end{array}\right]
$$

where all diagonal blocks are $p \times p$.

Set $U_{2} Q_{U}^{H}=:\left[P_{U}, P_{U}^{+}\right], W_{2} Q_{W}^{H}=:\left[P_{W}, P_{W}^{+}\right]$and take $V_{2} Q_{V}^{H}=:\left[0, P_{V}^{+}\right]$. The block forms of $\tilde{Z}, \tilde{T}$ and $\tilde{H}$ together with the first identity of (3.7) imply that $\bar{P}_{U} \tilde{Z}_{11}=\overline{P_{U}^{+}} \tilde{Z}_{21}$. Since the columns of $\left[\begin{array}{l}U_{1} \\ U_{2}\end{array}\right]$ are orthonormal, we have $\left(P_{U}^{+}\right)^{H} P_{U}^{+}=I_{p}$ and $\left(P_{U}^{+}\right)^{H} P_{U}=0$. Hence, $\tilde{Z}_{21}=0$, 
and consequently $P_{U}=0$. Similarly, from the third identity of (3.7) we get $P_{W}=0, \tilde{H}_{21}=0$ and from the second identity we obtain $\tilde{T}_{21}=0$. Combining all these observations, we obtain

$$
\begin{aligned}
& {\left[\begin{array}{cc}
\mathcal{Z} & 0 \\
0 & \overline{\mathcal{Z}}
\end{array}\right]\left[\begin{array}{cc}
P_{V}^{-} & 0 \\
0 & P_{V}^{+}
\end{array}\right]=\left[\begin{array}{cc}
P_{U}^{-} & 0 \\
0 & P_{U}^{+}
\end{array}\right]\left[\begin{array}{cc}
\tilde{Z}_{11} & 0 \\
0 & \tilde{Z}_{22}
\end{array}\right],} \\
& {\left[\begin{array}{cc}
\mathcal{J Z}^{H} \mathcal{J}^{T} & 0 \\
0 & \mathcal{J Z}^{H} \mathcal{J}^{T}
\end{array}\right]\left[\begin{array}{cc}
P_{U}^{-} & 0 \\
0 & P_{U}^{+}
\end{array}\right]=\left[\begin{array}{cc}
P_{W}^{-} & 0 \\
0 & P_{W}^{+}
\end{array}\right]\left[\begin{array}{cc}
\tilde{T}_{11} & 0 \\
0 & \tilde{T}_{22}
\end{array}\right],} \\
& {\left[\begin{array}{cc}
\mathcal{H} & 0 \\
0 & -\overline{\mathcal{H}}
\end{array}\right]\left[\begin{array}{cc}
P_{V}^{-} & 0 \\
0 & P_{V}^{+}
\end{array}\right]=\left[\begin{array}{cc}
P_{W}^{-} & 0 \\
0 & P_{W}^{+}
\end{array}\right]\left[\begin{array}{cc}
\tilde{H}_{11} & 0 \\
0 & \tilde{H}_{22}
\end{array}\right],}
\end{aligned}
$$

which gives (3.3).

We remark that (3.1) can be constructed from (2.25) by reordering the eigenvalues properly.

Theorem 3.1 gives a way to obtain the stable deflating subspace of a skew-Hamiltonian/Hamiltonian matrix pencil from the deflating subspaces of an embedded skew-Hamiltonian/Hamiltonian matrix pencil of double size. This will be used by the algorithms formulated in the next section.

4. Algorithms. The results of Theorem 3.1 together with the embedding technique lead to the following algorithm to compute the eigenvalues and the deflating subspaces $\operatorname{Def}_{-}(\mathcal{S}, \mathcal{H})$ and $\operatorname{Def}_{+}(\mathcal{S}, \mathcal{H})$ of a complex skew-Hamiltonian/Hamiltonian matrix pencil $\alpha \mathcal{S}-\beta \mathcal{H}$. Since the algorithms are rather technical, we do not discuss details like eigenvalue reordering or explicit elimination orders in the construction of the structured Schur forms. Instead we refer the reader to the technical report [4] for these details.

In summary, Algorithm 1 proposed below transforms a $2 n \times 2 n$ complex skew-Hamiltonian/Hamiltonian matrix pencil with $\mathcal{J}$-semidefinite skew-Hamiltonian part into a $4 n \times 4 n$ complex skewHamiltonian/Hamiltonian matrix pencil in Schur form. The process passes through intermediate matrix pencils of the following types.

$$
\begin{gathered}
2 n \times 2 n \text { complex skew-Hamiltonian/Hamiltonian matrix pencil } \\
\alpha \mathcal{S}-\beta \mathcal{H} \text { with } \mathcal{S}=\mathcal{J Z}^{H} \mathcal{J}^{T} \mathcal{Z} . \\
\Downarrow \\
\text { Equation } \\
\Downarrow
\end{gathered}
$$

$4 n \times 4 n$ real skew-Hamiltonian/skew-Hamiltonian matrix pencil

$$
\alpha \mathcal{B}_{\mathcal{S}}^{c}-\beta \mathcal{B}_{\mathcal{N}}^{c} \text { with } \mathcal{B}_{\mathcal{S}}^{c}=\mathcal{J}\left(\mathcal{B}_{\mathcal{Z}}^{c}\right)^{T} \mathcal{J}^{T} \mathcal{B}_{\mathcal{Z}}^{c}
$$

Algorithm 3 in [4]

$$
\Downarrow
$$

$4 n \times 4 n$ real skew-Hamiltonian/skew-Hamiltonian matrix pencil in Schur form

$$
\begin{gathered}
\alpha \tilde{\mathcal{B}}_{\mathcal{S}}^{c}-\beta \tilde{\mathcal{B}}_{\mathcal{N}}^{c} \text { with } \tilde{\mathcal{B}}_{\mathcal{S}}^{c}=\mathcal{J}\left(\tilde{\mathcal{B}}_{\mathcal{Z}}^{c}\right)^{T} \mathcal{J}^{T} \tilde{\mathcal{B}}_{\mathcal{Z}}^{c} \\
\text { and } \tilde{\mathcal{B}}_{\mathcal{Z}}^{c}=\mathcal{U}^{T} \mathcal{B}_{\mathcal{Z}}^{c} \mathcal{Q}=\left[\begin{array}{cc}
\mathcal{Z}_{11} & \mathcal{Z}_{12} \\
0 & \mathcal{Z}_{22}
\end{array}\right], \tilde{\mathcal{B}}_{\mathcal{N}}^{c}=\mathcal{J} \mathcal{Q}^{T} \mathcal{J}^{T} \mathcal{B}_{\mathcal{N}}^{c} \mathcal{Q}=\left[\begin{array}{cc}
\mathcal{N}_{11} & \mathcal{N}_{12} \\
0 & \mathcal{N}_{11}^{T}
\end{array}\right] \text { as in }(2.19) \text { and }(2.20) \\
\Downarrow \\
\text { Algorithm } 4 \text { in }[4] \\
\Downarrow
\end{gathered}
$$

$4 n \times 4 n$ complex skew-Hamiltonian/Hamiltonian matrix pencil in Schur form with ordered eigenvalues.

The required deflating subspaces of the original skew-Hamiltonian/Hamiltonian matrix pencil are then obtained from the deflating subspaces of the final $4 n \times 4 n$ complex skew-Hamiltonian/Hamiltonian matrix pencil. (Unfortunately, if there are non-real eigenvalues, then Algorithm 4 in [4] (the eigenvalue sorting algorithm) reintroduces complex entries into the $4 n \times 4 n$ extended real matrix pencil.)

Algorithm 1. Given a complex skew-Hamiltonian/Hamiltonian matrix pencil $\alpha \mathcal{S}-\beta \mathcal{H}$ with $\mathcal{J}$-semidefinite skew-Hamiltonian part $\mathcal{S}=\mathcal{J Z}^{H} \mathcal{J}^{T} \mathcal{Z}$, this algorithm computes the structured 
Schur form of the extended skew-Hamiltonian/Hamiltonian matrix pencil $\alpha \mathcal{B}_{\mathcal{S}}^{c}-\beta \mathcal{B}_{\mathcal{H}}^{c}$, the eigenvalues of $\alpha \mathcal{S}-\beta \mathcal{H}$, and orthonormal bases of the deflating subspace $\operatorname{Def}_{-}(\mathcal{S}, \mathcal{H})$ and the companion subspace range $P_{U}^{-}$.

Input: Hamiltonian matrix $\mathcal{H}$ and the factor $\mathcal{Z}$ of $\mathcal{S}$.

Output: $P_{V}^{-}, P_{U}^{-}$as defined in Theorem 3.1.

\section{Step 1:}

Set $\mathcal{N}=i \mathcal{H}$ and form matrices $\mathcal{B}_{\mathcal{Z}}^{c}, \mathcal{B}_{\mathcal{N}}^{c}$ as in (2.16) and (2.12), respectively. Find the structured Schur form of the skew-Hamiltonian/skew-Hamiltonian matrix pencil $\alpha \mathcal{B}_{\mathcal{S}}^{c}-$ $\beta \mathcal{B}_{\mathcal{N}}^{c}$ using Algorithm 3 in [4] to compute the factorization

$$
\begin{aligned}
\tilde{\mathcal{B}}_{\mathcal{Z}}^{c} & =\mathcal{U}^{T} \mathcal{B}_{\mathcal{Z}}^{c} \mathcal{Q}=\left[\begin{array}{cc}
\mathcal{Z}_{11} & \mathcal{Z}_{12} \\
0 & \mathcal{Z}_{22}
\end{array}\right] \\
\tilde{\mathcal{B}}_{\mathcal{N}}^{c} & =\mathcal{J} \mathcal{Q}^{T} \mathcal{J}^{T} \mathcal{B}_{\mathcal{N}}^{c} \mathcal{Q}=\left[\begin{array}{cc}
\mathcal{N}_{11} & \mathcal{N}_{12} \\
0 & \mathcal{N}_{11}^{T}
\end{array}\right],
\end{aligned}
$$

where $\mathcal{Q}$ is real orthogonal, $\mathcal{U}$ is real orthogonal symplectic, $\mathcal{Z}_{11}, \mathcal{Z}_{22}^{T}$ are upper triangular

Step 2: and $\mathcal{N}_{11}$ is quasi upper triangular.

Reorder the eigenvalues using Algorithm 4 in [4] to determine a unitary matrix $\tilde{\mathcal{Q}}$ and a unitary symplectic matrix $\tilde{\mathcal{U}}$ such that

$$
\begin{aligned}
\tilde{\mathcal{U}}^{H} \tilde{\mathcal{B}}_{\mathcal{Z}}^{c} \tilde{\mathcal{Q}} & =\left[\begin{array}{cc}
\tilde{\mathcal{Z}}_{11} & \tilde{\mathcal{Z}}_{12} \\
0 & \tilde{\mathcal{Z}}_{22}
\end{array}\right]=: \check{\mathcal{B}}_{\mathcal{Z}}^{c}, \\
\mathcal{J} \tilde{\mathcal{Q}}^{H} \mathcal{J}^{T}\left(-i \tilde{\mathcal{B}}_{\mathcal{N}}^{c}\right) \tilde{\mathcal{Q}} & =\left[\begin{array}{cc}
\mathcal{H}_{11} & \mathcal{H}_{12} \\
0 & -\mathcal{H}_{11}^{H}
\end{array}\right]=: \check{\mathcal{B}}_{\mathcal{H}}^{c},
\end{aligned}
$$

with $\tilde{\mathcal{Z}}_{11}, \tilde{\mathcal{Z}}_{22}^{H}, \mathcal{H}_{11}$ upper triangular such that $\Lambda_{-}\left(\mathcal{J}\left(\check{\mathcal{B}}_{\mathcal{Z}}^{c}\right)^{H} \tilde{J}^{T} \check{\mathcal{B}}_{\mathcal{Z}}^{c}, \check{\mathcal{B}}_{\mathcal{H}}^{c}\right)$ is contained in Step 3: the spectrum of the $2 p \times 2 p$ leading principal sub-pencil of $\alpha \tilde{\mathcal{Z}}_{22}^{H} \tilde{\mathcal{Z}}_{11}-\beta \mathcal{H}_{11}$.

Set $V=\left[I_{2 n}, 0\right] \mathcal{X}_{c} \mathcal{Q} \tilde{\mathcal{Q}}\left[\begin{array}{c}I_{2 p} \\ 0\end{array}\right], U=\left[I_{2 n}, 0\right] \mathcal{X}_{c} \mathcal{U} \tilde{\mathcal{U}}\left[\begin{array}{c}I_{2 p} \\ 0\end{array}\right]$ (where $\mathcal{X}_{c}$ is as in (2.11)) and compute $P_{V}^{-}, P_{U}^{-}$, orthogonal bases of range $V$ and range $U$, respectively, using any numerically stable orthogonalization scheme.

End

Based on flop counts, we estimate the cost of this algorithm to be roughly $50 \%$ of the cost of the periodic $Q Z$ algorithm [10,19] applied to the $2 n \times 2 n$ complex pencil $\alpha \mathcal{J Z}^{H} \mathcal{J}^{T} \mathcal{Z}-\beta \mathcal{H}$ (treating $\mathcal{J} \mathcal{Z}^{H} \mathcal{J}^{T}$ as one matrix).

If $\mathcal{S}$ is not factored, then the algorithm can be simplified by using the method of [32] to compute the real skew-Hamiltonian/Hamiltonian Schur form of $\alpha \mathcal{B}_{\mathcal{S}}^{c}-\beta \mathcal{B}_{\mathcal{H}}^{c}$ directly.

Algorithm 2. Given a complex skew-Hamiltonian/Hamiltonian matrix pencil $\alpha \mathcal{S}-\beta \mathcal{H}$. This algorithm computes the structured Schur form of the extended skew-Hamiltonian/Hamiltonian matrix pencil $\alpha \mathcal{B}_{\mathcal{S}}^{c}-\beta \mathcal{B}_{\mathcal{H}}^{c}$, the eigenvalues of $\alpha \mathcal{S}-\beta \mathcal{H}$, and an orthogonal basis of the deflating subspace Def $_{-}(\mathcal{S}, \mathcal{H})$.

Input: A complex skew-Hamiltonian/Hamiltonian matrix pencil $\alpha \mathcal{S}-\beta \mathcal{H}$.

Output: $P_{V}^{-}$as defined in Theorem 3.1.

Step 1:

Set $\mathcal{N}=i \mathcal{H}$ and form the matrices $\mathcal{B}_{\mathcal{S}}^{c}, \mathcal{B}_{\mathcal{N}}^{c}$ as in (2.17) and (2.12), respectively.

Find the structured Schur form of the skew-Hamiltonian/skew-Hamiltonian matrix pencil $\alpha \mathcal{B}_{\mathcal{S}}^{c}-\beta \mathcal{B}_{\mathcal{N}}^{c}$ using Algorithm 5 in [4] to compute the factorization

$$
\begin{aligned}
& \check{\mathcal{B}}_{\mathcal{S}}^{c}=\mathcal{J} \mathcal{Q}^{T} \mathcal{J}^{T} \mathcal{B}_{\mathcal{S}}^{c} \mathcal{Q}=\left[\begin{array}{cc}
\mathcal{S}_{11} & \mathcal{S}_{12} \\
0 & \mathcal{S}_{11}^{T}
\end{array}\right], \\
& \check{\mathcal{B}}_{\mathcal{N}}^{c}=\mathcal{J} \mathcal{Q}^{T} \mathcal{J}^{T} \mathcal{B}_{\mathcal{N}}^{c} \mathcal{Q}=\left[\begin{array}{cc}
\mathcal{N}_{11} & \mathcal{N}_{12} \\
0 & \mathcal{N}_{11}^{T}
\end{array}\right],
\end{aligned}
$$

where $\mathcal{Q}$ is real orthogonal, $\mathcal{S}_{11}$ is upper triangular and $\mathcal{N}_{11}$ is quasi upper triangular. 


\section{Step 2:}

Reorder the eigenvalues using Algorithm 6 in [4] to determine a unitary matrix $\tilde{\mathcal{Q}}$ such that

$$
\begin{aligned}
\mathcal{J} \tilde{\mathcal{Q}}^{H} \mathcal{J}^{T} \check{\mathcal{B}}_{\mathcal{S}}^{c} \tilde{\mathcal{Q}} & =\left[\begin{array}{cc}
\tilde{\mathcal{S}}_{11} & \tilde{\mathcal{S}}_{12} \\
0 & \tilde{\mathcal{S}}_{11}^{H}
\end{array}\right], \\
\mathcal{J} \tilde{\mathcal{Q}}^{H} \mathcal{J}^{T}\left(-i \check{\mathcal{B}}_{\mathcal{N}}^{c}\right) \tilde{\mathcal{Q}} & =\left[\begin{array}{cc}
\mathcal{H}_{11} & \mathcal{H}_{12} \\
0 & -\mathcal{H}_{11}^{H}
\end{array}\right],
\end{aligned}
$$

with $\tilde{\mathcal{S}}_{11}, \mathcal{H}_{11}$ upper triangular and such that $\Lambda_{-}\left(\check{\mathcal{B}}_{\mathcal{S}}^{c},-i \check{\mathcal{B}}_{\mathcal{N}}^{c}\right)$ is contained in the spectrum Step 3: of the $2 p \times 2 p$ leading principal sub-pencil of $\alpha \tilde{\mathcal{S}}_{11}-\beta \mathcal{H}_{11}$.

Set $V=\left[I_{2 n}, 0\right] \mathcal{X}_{c} \mathcal{Q} \tilde{\mathcal{Q}}\left[\begin{array}{c}I_{2 p} \\ 0\end{array}\right]$ (where $\mathcal{X}_{c}$ is as in (2.11)) and compute $P_{V}^{-}$, the orthogonal End

basis of range $V$, using any numerically stable orthogonalization scheme.

Algorithm 2 needs roughly $80 \%$ of the $1600 n^{3}$ real flops required by the $Q Z$ algorithm applied to the $2 n \times 2 n$ complex pencil $\alpha \mathcal{S}-\beta \mathcal{H}$ as suggested in [37]. If only the eigenvalues are computed, then Algorithm 2 without accumulation of $V$ needs roughly $60 \%$ of the $960 n^{3}$ real flops required by the $Q Z$ algorithm.

In this section we have presented numerical algorithms for the computation of (complex) structured triangular forms. Various details appear in [4]. In the next section we give an error analysis. The analysis is a generalization of the analysis for Hamiltonian matrices in $[6,7,8]$.

5. Error and Perturbation Analysis. In this section we will give the perturbation analysis for eigenvalues and deflating subspaces of skew-Hamiltonian/Hamiltonian matrix pencils. Variables marked with a circumflex denote perturbed quantities.

We begin with the perturbation analysis for the eigenvalues of $\alpha \mathcal{S}-\beta \mathcal{H}$ and $\alpha \mathcal{J Z}^{H} \mathcal{J}^{T} \mathcal{Z}-\beta \mathcal{H}$. In principle, we could multiply out $\mathcal{J Z}^{H} \mathcal{J}^{T} \mathcal{Z}$ and apply the classical perturbation analysis of matrix pencils using the chordal metric [36], but this may give pessimistic bounds and would display neither the effects of perturbing each factor separately nor the effects of structured perturbations. Therefore, we make use of the perturbation analysis for formal products of matrices developed in [9].

If Algorithm 2 is applied to the skew-Hamiltonian/Hamiltonian matrix pencil $\alpha \mathcal{S}-\beta \mathcal{H}$, then we compute the structured Schur form of the extended skew-Hamiltonian/Hamiltonian matrix pencil $\alpha \mathcal{B}_{\mathcal{S}}^{c}-\beta \mathcal{B}_{\mathcal{H}}^{c}$. The well-known backward error analysis of orthogonal matrix computations implies that rounding errors in Algorithm 2 are equivalent to perturbing $\alpha \mathcal{B}_{\mathcal{S}}^{c}-\beta \mathcal{B}_{\mathcal{H}}^{c}$ to a nearby matrix pencil $\alpha \hat{\mathcal{B}}_{\mathcal{S}}^{c}-\beta \hat{\mathcal{B}}_{\mathcal{H}}^{c}$, where

$$
\begin{aligned}
& \hat{\mathcal{B}}_{\mathcal{S}}^{c}=\mathcal{B}_{\mathcal{S}}^{c}+\mathcal{E}_{\mathcal{S}}, \\
& \hat{\mathcal{B}}_{\mathcal{H}}^{c}=\mathcal{B}_{\mathcal{H}}^{c}+\mathcal{E}_{\mathcal{H}},
\end{aligned}
$$

with $\mathcal{E}_{\mathcal{S}} \in \mathbb{S H}_{4 n}, \mathcal{E}_{\mathcal{H}} \in \mathbb{H}_{4 n}$ and

$$
\begin{gathered}
\left\|\mathcal{E}_{\mathcal{S}}\right\|_{2}<c_{\mathcal{S}} \varepsilon\left\|\mathcal{B}_{\mathcal{S}}^{c}\right\|_{2}, \\
\left\|\mathcal{E}_{\mathcal{H}}\right\|_{2}<c_{\mathcal{H}} \varepsilon\left\|\mathcal{B}_{\mathcal{H}}^{c}\right\|_{2}
\end{gathered}
$$

Here $\varepsilon$ is the unit round of the floating point arithmetic and $c_{\mathcal{S}}$ and $c_{\mathcal{H}}$ are modest constants depending on the details of the implementation and arithmetic. Let $x$ and $y$ be unit norm vectors such that

$$
\mathcal{H} x=\alpha_{1} y, \quad \mathcal{S} x=\beta_{1} y
$$

and let $\lambda=\alpha_{1} / \beta_{1}$ be a simple eigenvalue of $\alpha \mathcal{S}-\beta \mathcal{H}$. If $\lambda$ is finite and $\operatorname{Re} \lambda \neq 0$, then $-\bar{\lambda}$ is also a simple eigenvalue of $\alpha \mathcal{S}-\beta \mathcal{H}$. Let $u, v$ be unit norm vectors such that

$$
\mathcal{H} u=\alpha_{2} v, \quad \mathcal{S} u=\beta_{2} v
$$


and $\alpha_{2} / \beta_{2}=-\bar{\lambda}$. Then we have

$$
-\overline{\mathcal{H}} \bar{u}=-\bar{\alpha}_{2} \bar{v}, \quad \overline{\mathcal{S}} \bar{u}=\bar{\beta}_{2} \bar{v} .
$$

Using the equivalence of the matrix pencils $\alpha \mathcal{B}_{\mathcal{S}}^{c}-\beta \mathcal{B}_{\mathcal{H}}^{c}$ and $\alpha \mathcal{B}_{\mathcal{S}}-\beta \mathcal{B}_{\mathcal{H}}$, and setting

$$
\mathcal{U}_{1}=\mathcal{X}_{c}^{H}\left[\begin{array}{ll}
y & 0 \\
0 & \bar{v}
\end{array}\right], \quad \mathcal{U}_{2}=\mathcal{X}_{c}^{H}\left[\begin{array}{cc}
x & 0 \\
0 & \bar{u}
\end{array}\right]
$$

we obtain from (5.5) and (5.7) that

$$
\mathcal{B}_{\mathcal{H}}^{c} \mathcal{U}_{2}=\mathcal{U}_{1}\left[\begin{array}{cc}
\alpha_{1} & 0 \\
0 & -\bar{\alpha}_{2}
\end{array}\right], \quad \mathcal{B}_{\mathcal{S}}^{c} \mathcal{U}_{2}=\mathcal{U}_{1}\left[\begin{array}{cc}
\beta_{1} & 0 \\
0 & \bar{\beta}_{2}
\end{array}\right],
$$

which implies that $\lambda$ is a double eigenvalue of $\alpha \mathcal{B}_{\mathcal{S}}^{c}-\beta \mathcal{B}_{\mathcal{H}}^{c}$ with a complete set of linearly independent eigenvectors. Similarly, $-\bar{\lambda}$ is a double eigenvalue of $\alpha \mathcal{B}_{\mathcal{S}}^{c}-\beta \mathcal{B}_{\mathcal{H}}^{c}$ with a complete set of linearly independent eigenvectors and

$$
\mathcal{B}_{\mathcal{H}}^{c} \mathcal{V}_{2}=\mathcal{V}_{1}\left[\begin{array}{cc}
\alpha_{2} & 0 \\
0 & -\bar{\alpha}_{1}
\end{array}\right], \quad \mathcal{B}_{\mathcal{S}}^{c} \mathcal{V}_{2}=\mathcal{V}_{1}\left[\begin{array}{cc}
\beta_{2} & 0 \\
0 & \bar{\beta}_{1}
\end{array}\right]
$$

where

$$
\mathcal{V}_{1}=\mathcal{X}_{c}^{H}\left[\begin{array}{ll}
v & 0 \\
0 & \bar{y}
\end{array}\right], \quad \mathcal{V}_{2}=\mathcal{X}_{c}^{H}\left[\begin{array}{cc}
u & 0 \\
0 & \bar{x}
\end{array}\right]
$$

Note that the finite eigenvalues with non-zero real part appear in pairs as in (5.5) and (5.6), but infinite and purely imaginary eigenvalues may not appear in pairs. Consequently, in the following perturbation theorem, the bounds for purely imaginary and infinite eigenvalues are different from the bounds for finite eigenvalues with non-zero real part.

THEOREM 5.1. Consider the skew-Hamiltonian/Hamiltonian matrix pencil $\alpha \mathcal{S}-\beta \mathcal{H}$ along with the corresponding extended matrix pencils $\alpha \mathcal{B}_{\mathcal{S}}^{c}-\beta \mathcal{B}_{\mathcal{H}}^{c}=\mathcal{X}_{c}^{H}\left(\alpha \mathcal{B}_{\mathcal{S}}-\beta \mathcal{B}_{\mathcal{H}}\right) \mathcal{X}_{c}$, where $\mathcal{B}_{\mathcal{S}}$ is given by (2.15), $\mathcal{B}_{\mathcal{H}}$ by (2.21), $\mathcal{B}_{\mathcal{H}}^{c}$ by (2.22), $\mathcal{X}_{c}$ by (2.11) and $\mathcal{B}_{\mathcal{S}}^{c}$ by (2.17). Let $\alpha \hat{\mathcal{B}}_{\mathcal{S}}^{c}-\beta \hat{\mathcal{B}}_{\mathcal{H}}^{c}$ be a perturbed extended matrix pencil satisfying (5.1)-(5.4) with constants $c_{\mathcal{H}}, c_{\mathcal{S}}$ and let $\varepsilon$ be equal to the unit round of the floating point arithmetic.

If $\lambda$ is a simple eigenvalue of $\alpha \mathcal{S}-\beta \mathcal{H}$ with vectors $x$ and $y$ as in (5.5) and vectors $u$ and $v$ as in (5.6), then the corresponding double eigenvalue of $\alpha \mathcal{B}_{\mathcal{S}}^{c}-\beta \mathcal{B}_{\mathcal{H}}^{c}$ may split into two eigenvalues $\hat{\lambda}_{1}$ and $\hat{\lambda}_{2}$ of the perturbed matrix pencil $\alpha \hat{\mathcal{B}}_{\mathcal{S}}^{c}-\beta \hat{\mathcal{B}}_{\mathcal{H}}^{c}$, each of which satisfies the following bounds.

(i) If $\lambda$ is finite and $\operatorname{Re} \lambda \neq 0$, then

$$
\left|\frac{\hat{\lambda}_{k}-\lambda}{\lambda}\right| \leq \frac{\varepsilon}{\left|u^{H} \mathcal{J} y\right|}\left(\frac{c_{\mathcal{H}}}{\left|\alpha_{1}\right|}\|\mathcal{H}\|_{2}+\frac{c_{\mathcal{S}}}{\left|\beta_{1}\right|}\|\mathcal{S}\|_{2}\right)+O\left(\varepsilon^{2}\right), \quad k=1,2 .
$$

(ii) If $\lambda$ is finite and $\operatorname{Re} \lambda=0$, then

$$
\left|\hat{\lambda}_{k}-\lambda\right| \leq \frac{\varepsilon}{\left|\beta_{1}\right|\left|x^{H} \mathcal{J} y\right|}\left(c_{\mathcal{H}}\|\mathcal{H}\|_{2}+c_{\mathcal{S}}|\lambda|\|\mathcal{S}\|_{2}\right)+O\left(\varepsilon^{2}\right), \quad k=1,2 .
$$

(iii) If $\lambda=\infty$, then

$$
\frac{1}{\left|\hat{\lambda}_{k}\right|} \leq \varepsilon \frac{c_{\mathcal{S}}\|\mathcal{S}\|_{2}}{\left|\alpha_{1}\right|\left|x^{H} \mathcal{J} y\right|}+O\left(\varepsilon^{2}\right), \quad k=1,2 .
$$

Proof. We first consider the case that $\lambda$ is finite and $\operatorname{Re} \lambda \neq 0$. Let $\mathcal{U}_{1}$ and $\mathcal{U}_{2}$ be defined by (5.8) and $\mathcal{V}_{1}$ and $\mathcal{V}_{2}$ be defined by (5.9). Using the perturbation theory for formal products of matrices (see [9]), we obtain

$$
\begin{aligned}
\left|\frac{\hat{\lambda}_{k}-\lambda}{\lambda}\right| \leq \min \left(\left\|\left(\mathcal{V}_{2}^{H} \mathcal{J} \mathcal{U}_{1} C_{\mathcal{S}}\right)^{-1} \mathcal{V}_{2}^{H} \mathcal{J}\left(\frac{1}{\lambda} \mathcal{E}_{\mathcal{H}}-\mathcal{E}_{\mathcal{S}}\right) \mathcal{U}_{2}\right\|_{2},\right. \\
\left.\left\|\left(\mathcal{V}_{2}^{H} \mathcal{J} \mathcal{U}_{1}\right)^{-1} \mathcal{V}_{2}^{H} \mathcal{J}\left(\frac{1}{\lambda} \mathcal{E}_{\mathcal{H}}-\mathcal{E}_{\mathcal{S}}\right) \mathcal{U}_{2} C_{\mathcal{S}}^{-1}\right\|_{2}\right)+O\left(\varepsilon^{2}\right) .
\end{aligned}
$$


Here, $C_{\mathcal{S}}=\left[\begin{array}{cc}\beta_{1} & 0 \\ 0 & \bar{\beta}_{2}\end{array}\right]$ and $\mathcal{V}_{2}^{H} \mathcal{J} \mathcal{U}_{1}=\left[\begin{array}{cc}u & 0 \\ 0 & \bar{x}\end{array}\right]^{H} \mathcal{X}_{c} \mathcal{J} \mathcal{X}_{c}^{H}\left[\begin{array}{cc}y & 0 \\ 0 & \bar{v}\end{array}\right]=\left[\begin{array}{cc}u^{H} \mathcal{J} y & 0 \\ 0 & x^{T} \mathcal{J}_{\bar{v}}\end{array}\right]$. The second equation in (5.6) implies $u^{H} \mathcal{J S}=\bar{\beta}_{2} v^{H} \mathcal{J}$. Combining this with the second equation of (5.5) we get $\bar{\beta}_{2} v^{H} \mathcal{J} x=\beta_{1} u^{H} \mathcal{J} y$. Hence,

$$
\begin{aligned}
\left|\frac{\hat{\lambda}_{k}-\lambda}{\lambda}\right| & \leq\left\|\left(\mathcal{V}_{2}^{H} \mathcal{J} \mathcal{U}_{1} C_{\mathcal{S}}\right)^{-1} \mathcal{V}_{2}^{H} \mathcal{J}\left(\frac{1}{\lambda} \mathcal{E}_{\mathcal{H}}-\mathcal{E}_{\mathcal{S}}\right) \mathcal{U}_{2}\right\|_{2}+O\left(\varepsilon^{2}\right) \\
& \leq\left\|\left(\mathcal{V}_{2}^{H} \mathcal{J} \mathcal{U}_{1} C_{\mathcal{S}}\right)^{-1}\right\|_{2}\left\|\frac{1}{\lambda} \mathcal{E}_{\mathcal{H}}-\mathcal{E}_{\mathcal{S}}\right\|_{2}+O\left(\varepsilon^{2}\right) \\
& \leq \frac{1}{\left|u^{H} \mathcal{J} y\right|}\left(\frac{\left\|\mathcal{E}_{\mathcal{H}}\right\|_{2}}{\left|\beta_{1} \lambda\right|}+\frac{\left\|\mathcal{E}_{\mathcal{S}}\right\|_{2}}{\left|\beta_{1}\right|}\right)+O\left(\varepsilon^{2}\right) \\
& \leq \frac{\varepsilon}{\left|u^{H} \mathcal{J} y\right|}\left(\frac{c_{\mathcal{H}}}{\left|\alpha_{1}\right|}\|\mathcal{H}\|_{2}+\frac{c_{\mathcal{S}}}{\left|\beta_{1}\right|}\|\mathcal{S}\|_{2}\right)+O\left(\varepsilon^{2}\right) .
\end{aligned}
$$

If $\lambda$ is purely imaginary or infinite, then the bounds are obtained by adapting the classical perturbation theory in [36] to a formal product of matrices (for details see [9]) and by replacing (5.7) with $-\overline{\mathcal{H}} \bar{x}=-\bar{\alpha}_{1} \bar{y}$ and $\overline{\mathcal{S}} \bar{x}=\bar{\beta}_{1} \bar{y}$ as well as replacing $u, v, \alpha_{2}$ and $\beta_{2}$ by $x, y, \alpha_{1}$ and $\beta_{1}$, respectively.

The bound in part 1 appears to involve only $u, y, \alpha_{1}$ and $\beta_{1}$ but not $v, x, \alpha_{2}$ and $\beta_{2}$. However, note in the proof that $\bar{\beta}_{2} v^{H} \mathcal{J} x=\beta_{1} u^{H} \mathcal{J} y$, so the bound implicitly involves all the parameters. Note further, that if $\mathcal{S}$ is nonsingular, then $v^{H} \mathcal{J} x$ and $u^{H} \mathcal{J} y$ are just the reciprocals of the condition number of $\lambda$ as eigenvalue of $\mathcal{S}^{-1} \mathcal{H}$ and $\mathcal{H} \mathcal{S}^{-1}$, respectively, see [6].

If $\mathcal{S}$ is given in factored form, Algorithm 1 computes a unitary symplectic matrix $\mathcal{U}$ and a unitary matrix $\mathcal{Q}$ which reduce the perturbed matrices

$$
\hat{\mathcal{B}}_{\mathcal{Z}}^{c}:=\mathcal{B}_{\mathcal{Z}}^{c}+\mathcal{E}_{\mathcal{Z}}, \quad \hat{\mathcal{B}}_{\mathcal{H}}^{c}:=\mathcal{B}_{\mathcal{H}}^{c}+\mathcal{E}_{\mathcal{H}}
$$

to block upper triangular form as in (2.23) and (2.24), where

$$
\left\|\mathcal{E}_{\mathcal{Z}}\right\|_{2} \leq c_{\mathcal{Z}} \varepsilon\left\|\mathcal{B}_{\mathcal{Z}}^{c}\right\|_{2}, \quad\left\|\mathcal{E}_{\mathcal{H}}\right\|_{2} \leq c_{\mathcal{H}} \varepsilon\left\|\mathcal{B}_{\mathcal{H}}^{c}\right\|_{2}
$$

and $c_{\mathcal{Z}}$ and $c_{\mathcal{H}}$ are constants. The eigenvalue perturbation bounds then are essentially the same as in Theorem 5.1.

THEOREM 5.2. Consider the skew-Hamiltonian/Hamiltonian matrix pencil $\alpha \mathcal{S}-\beta \mathcal{H}$ with $\mathcal{J}$-semidefinite skew-Hamiltonian part $\mathcal{S}=\mathcal{J Z}^{H} \mathcal{J}^{T} \mathcal{Z}$. Let $\alpha \mathcal{B}_{\mathcal{S}}^{c}-\beta \mathcal{B}_{\mathcal{H}}^{c}=\mathcal{X}_{c}^{H}\left(\alpha \mathcal{B}_{\mathcal{S}}-\beta \mathcal{B}_{\mathcal{H}}\right) \mathcal{X}_{c}$ be the corresponding extended matrix pencils, where $\mathcal{B}_{\mathcal{S}}^{c}=\mathcal{J}\left(\mathcal{B}_{\mathcal{Z}}^{c}\right)^{H} \mathcal{J}^{T} \mathcal{B}_{\mathcal{Z}}^{c}, \mathcal{B}_{\mathcal{Z}}$ and $\mathcal{B}_{\mathcal{Z}}^{c}$ are given by (2.13) and (2.16), $\mathcal{B}_{\mathcal{H}}$ and $\mathcal{B}_{\mathcal{H}}^{c}$ by (2.21) and (2.22), and $\mathcal{X}_{c}$ by (2.11). Let $\left(\hat{\mathcal{B}}_{\mathcal{Z}}^{c}\right.$, $\left.\hat{\mathcal{B}}_{\mathcal{H}}^{c}\right)$ be the perturbed extended matrix pair in (5.10), (5.11) with constants $c_{\mathcal{H}}, c_{\mathcal{Z}}$.

Let $\lambda$ be a simple eigenvalue of $\alpha \mathcal{S}-\beta \mathcal{H}=\alpha \mathcal{J} \mathcal{Z}^{H} \mathcal{J}^{T} \mathcal{Z}-\beta \mathcal{H}$ with $\operatorname{Re} \lambda \neq 0$, and let $x, y, z$, $u, v, w$ be unit norm vectors such that

$$
\mathcal{J Z}^{H} \mathcal{J}^{T} x=\alpha_{1} y, \quad \mathcal{H} z=\beta_{1} y, \quad \mathcal{Z} z=\gamma_{1} x
$$

with $\lambda=\frac{\beta_{1}}{\alpha_{1} \gamma_{1}}$, and

$$
\mathcal{J Z}^{H} \mathcal{J}^{T} u=\alpha_{2} v, \quad \mathcal{H} w=\beta_{2} v, \quad \mathcal{Z} w=\gamma_{2} u
$$

with $-\bar{\lambda}=\frac{\beta_{2}}{\alpha_{2} \gamma_{2}}$.

The corresponding double eigenvalue of $\alpha \mathcal{B}_{\mathcal{S}}^{c}-\beta \mathcal{B}_{\mathcal{H}}^{c}$ may split into two eigenvalues $\hat{\lambda}_{1}$ and $\hat{\lambda}_{2}$ of the perturbed matrix pencil $\alpha \hat{\mathcal{B}}_{\mathcal{S}}^{c}-\beta \hat{\mathcal{B}}_{\mathcal{H}}^{c}$, each of which satisfies the following bounds.

(i) If $\lambda$ is finite and $\operatorname{Re} \lambda \neq 0$, then

$$
\left|\frac{\hat{\lambda}_{k}-\lambda}{\lambda}\right| \leq \varepsilon\left(\frac{c_{\mathcal{H}}}{\left|\beta_{1} w^{H} \mathcal{J} y\right|}\|\mathcal{H}\|_{2}+2 \frac{c_{\mathcal{Z}}}{\min \left\{\left|\gamma_{1} u^{H} \mathcal{J} x\right|,\left|\alpha_{1} w^{H} \mathcal{J} y\right|\right\}}\|\mathcal{Z}\|_{2}\right)+O\left(\varepsilon^{2}\right)
$$


(ii) If $\lambda$ is purely imaginary, then

$$
\left|\hat{\lambda}_{k}-\lambda\right| \leq \varepsilon\left(\frac{c_{\mathcal{H}}}{\left|\alpha_{1} \gamma_{1} y^{H} \mathcal{J} z\right|}\|\mathcal{H}\|_{2}+\frac{2|\lambda| c_{\mathcal{Z}}}{\left|\gamma_{1} u^{H} \mathcal{J} x\right|}\|\mathcal{Z}\|_{2}\right)+O\left(\varepsilon^{2}\right) .
$$

(iii) If $\lambda=\infty$, then $\left|\hat{\lambda}_{k}\right|^{-1}=O\left(\varepsilon^{2}\right)$.

Proof. The perturbation analysis follows [9]. If $\lambda$ is finite and $\operatorname{Re} \lambda \neq 0$, then

$$
\left|\frac{\hat{\lambda}_{k}-\lambda}{\lambda}\right| \leq\left\|\left(\mathcal{V}_{2}^{H} \mathcal{J} \mathcal{U}_{3}\right)^{-1}\left(\tilde{C}_{1} \tilde{C}_{3}\right)^{-1}\left(\mathcal{V}_{3}^{H} \mathcal{E}_{\mathcal{Z}}^{H} \mathcal{J} \mathcal{U}_{1} C_{3}+\tilde{C}_{3}^{H} \mathcal{U}_{1}^{H} \mathcal{J} \mathcal{E}_{\mathcal{Z}} \mathcal{U}_{3}-\frac{1}{\lambda} \mathcal{V}_{3}^{H} \mathcal{J} \mathcal{E}_{\mathcal{H}} \mathcal{U}_{3}\right)\right\|_{2}+O\left(\varepsilon^{2}\right),
$$

where $\mathcal{U}_{1}=\mathcal{X}_{c}^{H}\left[\begin{array}{ll}x & 0 \\ 0 & \bar{u}\end{array}\right] \in \mathbb{C}^{4 n, 2}, \mathcal{U}_{3}=\mathcal{X}_{c}^{H}\left[\begin{array}{cc}z & 0 \\ 0 & \bar{w}\end{array}\right] \in \mathbb{C}^{4 n, 2}, \mathcal{V}_{2}=\mathcal{X}_{c}^{H}\left[\begin{array}{cc}v & 0 \\ 0 & \bar{y}\end{array}\right] \in \mathbb{C}^{4 n, 2}, \mathcal{V}_{3}=$ $\mathcal{X}_{c}^{H}\left[\begin{array}{ll}w & 0 \\ 0 & \bar{z}\end{array}\right] \in \mathbb{C}^{4 n, 2}$, and $\tilde{C}_{1}=\left[\begin{array}{cc}\alpha_{2} & 0 \\ 0 & \bar{\alpha}_{1}\end{array}\right] \in \mathbb{C}^{2,2}, \tilde{C}_{3}=\left[\begin{array}{cc}\gamma_{2} & 0 \\ 0 & \bar{\gamma}_{1}\end{array}\right] \in \mathbb{C}^{2,2}, C_{3}=\left[\begin{array}{cc}\gamma_{1} & 0 \\ 0 & \bar{\gamma}_{2}\end{array}\right] \in \mathbb{C}^{2,2}$.

From $\mathcal{V}_{2}^{H} \mathcal{J U}_{3}=\left[\begin{array}{cc}v^{H} \mathcal{J} z & 0 \\ 0 & y^{T} \mathcal{J} \bar{w}\end{array}\right]$, it follows that

$$
\left|\frac{\hat{\lambda}_{k}-\lambda}{\lambda}\right| \leq \frac{\max \left\{\left|\gamma_{1}\right|,\left|\gamma_{2}\right|\right\}\left\|\mathcal{E}_{\mathcal{Z}}\right\|_{2}+\frac{1}{|\lambda|}\left\|\mathcal{E}_{\mathcal{H}}\right\|_{2}}{\min \left\{\left|\bar{\alpha}_{2} \bar{\gamma}_{2} v^{H} \mathcal{J} z\right|,\left|\alpha_{1} \gamma_{1} w^{H} \mathcal{J} y\right|\right\}}+\frac{\left\|\mathcal{E}_{\mathcal{Z}}\right\|_{2}}{\min \left\{\left|\bar{\alpha}_{2} v^{H} \mathcal{J} z\right|,\left|\alpha_{1} w^{H} \mathcal{J} y\right|\right\}}+O\left(\varepsilon^{2}\right) .
$$

From (5.12) and (5.13), we also have

$$
\bar{\alpha}_{2} v^{H} \mathcal{J} z=\gamma_{1} u^{H} \mathcal{J} x, \quad \bar{\gamma}_{2} u^{H} \mathcal{J} x=\alpha_{1} w^{H} \mathcal{J} y, \quad \bar{\beta}_{2} v^{H} \mathcal{J} z=-\beta_{1} w^{H} \mathcal{J} y .
$$

It follows that

$$
\left|\bar{\alpha}_{2} \bar{\gamma}_{2} v^{H} \mathcal{J} z\right|=\left|\bar{\gamma}_{2} \gamma_{1} u^{H} \mathcal{J} x\right|=\left|\gamma_{1} \alpha_{1} w^{H} \mathcal{J} y\right| .
$$

Hence,

$$
\begin{aligned}
\frac{\max \left\{\left|\gamma_{1}\right|,\left|\gamma_{2}\right|\right\}}{\min \left\{\left|\bar{\alpha}_{2} \bar{\gamma}_{2} v^{H} \mathcal{J} z\right|,\left|\alpha_{1} \gamma_{1} w^{H} \mathcal{J} y\right|\right\}} & =\frac{1}{\min \left\{\left|\bar{\alpha}_{2} v^{H} \mathcal{J} z\right|,\left|\alpha_{1} w^{H} \mathcal{J} y\right|\right\}} \\
|\lambda| \min \left\{\left|\bar{\alpha}_{2} \bar{\gamma}_{2} v^{H} \mathcal{J} z\right|,\left|\alpha_{1} \gamma_{1} w^{H} \mathcal{J} y\right|\right\} & =\left|\beta_{1} w^{H} \mathcal{J} y\right|,
\end{aligned}
$$

and

$$
\left|\frac{\hat{\lambda}_{k}-\lambda}{\lambda}\right| \leq \varepsilon\left(\frac{c_{\mathcal{H}}}{\left|\beta_{1} w^{H} \mathcal{J} y\right|}\|\mathcal{H}\|_{2}+\frac{2 c_{\mathcal{Z}}}{\min \left\{\left|\bar{\alpha}_{2} v^{H} \mathcal{J} z\right|,\left|\alpha_{1} w^{H} \mathcal{J} y\right|\right\}}\|\mathcal{Z}\|_{2}\right)+O\left(\varepsilon^{2}\right) .
$$

Equation (5.14) implies that $\bar{\alpha}_{2} v^{H} \mathcal{J} z=\gamma_{1} u^{H} \mathcal{J} x$. The first part of the theorem follows.

If $\lambda$ is purely imaginary, the proof is analogous.

If $\lambda=\infty$, then $\alpha_{1}=0$ or $\gamma_{1}=0$ and $\beta_{1} \neq 0$. Using the first equation of (5.14), we have $\bar{\alpha}_{1} y^{H} \mathcal{J} z=\gamma_{1} x^{H} \mathcal{J} x$, where we have replaced $u, v$ and $\alpha_{2}$ by $x, y$ and $\alpha_{1}$, respectively ((5.12) and (5.13) are the same now). Since $\lambda$ is simple, i.e., $y^{H} \mathcal{J} z \neq 0$ and $x^{H} \mathcal{J} x \neq 0$, we have $\alpha_{1}=\gamma_{1}=0$ and hence,

$$
C_{1}=\left[\begin{array}{cc}
\alpha_{1} & 0 \\
0 & \bar{\alpha}_{1}
\end{array}\right]=0, \quad C_{3}=\left[\begin{array}{cc}
\gamma_{1} & 0 \\
0 & \bar{\gamma}_{1}
\end{array}\right]=0, \quad C_{2}=\left[\begin{array}{cc}
\beta_{1} & 0 \\
0 & \bar{\beta}_{1}
\end{array}\right] \neq 0 .
$$

Therefore,

$$
E_{\infty}:=C_{1}^{H} C_{2}^{-H} \mathcal{U}_{3}^{H} \mathcal{E}_{\mathcal{Z}}^{H} \mathcal{J U}_{1}-\mathcal{U}_{1}^{H} \mathcal{J E}_{\mathcal{Z}} \mathcal{U}_{3} C_{2}^{-1} C_{1}-C_{1}^{H} C_{2}^{-H} \mathcal{U}_{3}^{H} \mathcal{J E}_{\mathcal{H}} \mathcal{U}_{3} C_{2}^{-1} C_{1}=0
$$

From [9, Theorem 23, part b)], we get

$$
\left|\frac{1}{\hat{\lambda}_{k}}\right| \leq\left\|\left(\mathcal{U}_{1}^{H} \mathcal{J} \mathcal{U}_{1}\right)^{-1} E_{\infty}\right\|_{2}+O\left(\varepsilon^{2}\right)=O\left(\varepsilon^{2}\right) .
$$


If the matrix pencil $\alpha \mathcal{S}-\beta \mathcal{H}$ with $\mathcal{J}$-semidefinite skew-Hamiltonian part $\mathcal{S}=\mathcal{J Z}^{H} \mathcal{J}^{T} \mathcal{Z}$ has semi-simple, multiple, infinite eigenvalues, then the perturbation bound (5.15) weakens to $O(\varepsilon)$ $[9]$.

To study the perturbations in the computed deflating subspaces we need to study the perturbations for the extended matrix pencil in more detail. As mentioned before, by applying Algorithm 2 to $\alpha \mathcal{B}_{\mathcal{S}}^{c}-\beta \mathcal{B}_{\mathcal{H}}^{c}$ we actually compute a unitary matrix $\hat{\mathcal{Q}}$ such that

$$
\begin{aligned}
\mathcal{J} \hat{\mathcal{Q}}^{H} \mathcal{J}^{T}\left(\alpha \hat{\mathcal{B}}_{\mathcal{S}}^{c}-\beta \hat{\mathcal{B}}_{\mathcal{H}}^{c}\right) \hat{\mathcal{Q}}=\alpha \hat{\mathcal{R}}_{\mathcal{S}}-\beta \hat{\mathcal{R}}_{\mathcal{H}} \\
\quad=: \alpha\left[\begin{array}{cc}
\hat{\mathcal{S}}_{11} & \hat{\mathcal{S}}_{12} \\
0 & \hat{\mathcal{S}}_{11}^{H}
\end{array}\right]-\beta\left[\begin{array}{cc}
\hat{\mathcal{H}}_{11} & \hat{\mathcal{H}}_{12} \\
0 & -\hat{\mathcal{H}}_{11}^{H}
\end{array}\right],
\end{aligned}
$$

where $\hat{\mathcal{B}}_{\mathcal{S}}^{c}$ and $\hat{\mathcal{B}}_{\mathcal{H}}^{c}$ are defined in $(5.1)$ and $(5.2)$, and $\Lambda\left(\hat{\mathcal{S}}_{11}, \hat{\mathcal{H}}_{11}\right)=\Lambda_{-}\left(\hat{\mathcal{B}}_{\mathcal{S}}^{c}, \hat{\mathcal{B}}_{\mathcal{H}}^{c}\right)$. If we assume that the matrix pencil $\alpha \mathcal{S}-\beta \mathcal{H}$ has no purely imaginary eigenvalues, then by Theorem 2.6 there exist unitary matrices $\mathcal{Q}_{1}, \mathcal{Q}_{2}$ such that

$$
\mathcal{J} \mathcal{Q}_{1}^{H} \mathcal{J}^{T}(\alpha \mathcal{S}-\beta \mathcal{H}) \mathcal{Q}_{1}=\alpha\left[\begin{array}{cc}
S_{11}^{-} & S_{12}^{-} \\
0 & \left(S_{11}^{-}\right)^{H}
\end{array}\right]-\beta\left[\begin{array}{cc}
H_{11}^{-} & H_{12}^{-} \\
0 & -\left(H_{11}^{-}\right)^{H}
\end{array}\right]
$$

with $\Lambda\left(S_{11}^{-}, H_{11}^{-}\right)=\Lambda_{-}(\mathcal{S}, \mathcal{H})$, and

$$
\mathcal{J} \mathcal{Q}_{2}^{H} \mathcal{J}^{T}(\alpha \mathcal{S}-\beta \mathcal{H}) \mathcal{Q}_{2}=\alpha\left[\begin{array}{cc}
S_{11}^{+} & S_{12}^{+} \\
0 & \left(S_{11}^{+}\right)^{H}
\end{array}\right]-\beta\left[\begin{array}{cc}
H_{11}^{+} & H_{12}^{+} \\
0 & -\left(H_{11}^{+}\right)^{H}
\end{array}\right]
$$

with $\Lambda\left(S_{11}^{+}, H_{11}^{+}\right)=\Lambda_{+}(\mathcal{S}, \mathcal{H})$, respectively. Set $\mathcal{Q}=\mathcal{X}_{c}^{H} \operatorname{diag}\left(\mathcal{Q}_{1}, \overline{\mathcal{Q}}_{2}\right) \mathcal{P}$ with $\mathcal{P}$ and $\mathcal{X}_{c}$ as in (2.10) and (2.11). Then $\mathcal{Q}$ is unitary and

$$
\begin{aligned}
& \mathcal{J} \mathcal{Q}^{H} \mathcal{J}^{T}\left(\alpha \mathcal{B}_{\mathcal{S}}^{c}-\beta \mathcal{B}_{\mathcal{H}}^{c}\right) \mathcal{Q} \\
= & \alpha\left[\begin{array}{cc|cc}
S_{11}^{-} & 0 & S_{12}^{-} & \frac{0}{S_{12}^{+}} \\
0 & \overline{S_{11}^{+}} & 0 & 0 \\
\hline 0 & 0 & \left(S_{11}^{-}\right)^{H} & 0 \\
0 & 0 & 0 & \left(\overline{S_{11}^{+}}\right)^{H}
\end{array}\right]-\beta\left[\begin{array}{ccccc}
H_{11}^{-} & 0 & H_{12}^{-} & 0 \\
0 & -\overline{H_{11}^{+}} & 0 & -\overline{H_{12}^{+}} \\
\hline 0 & 0 & -\left(H_{11}^{-}\right)^{H} & 0 \\
0 & 0 & 0 & \left(\overline{H_{11}^{+}}\right)^{H}
\end{array}\right] \\
(5.17)= & \alpha\left[\begin{array}{cc}
\mathcal{S}_{11} & \mathcal{S}_{12} \\
0 & \mathcal{S}_{11}^{H}
\end{array}\right]-\beta\left[\begin{array}{cc}
\mathcal{H}_{11} & \mathcal{H}_{12} \\
0 & -\mathcal{H}_{11}^{H}
\end{array}\right] \\
= & \alpha \mathcal{R}_{\mathcal{S}}-\beta \mathcal{R}_{\mathcal{H}} .
\end{aligned}
$$

This is the structured Schur form of the extended skew-Hamiltonian/Hamiltonian matrix pencil $\alpha \mathcal{B}_{\mathcal{S}}^{c}-\beta \mathcal{B}_{\mathcal{H}}^{c}$. Moreover, $\Lambda\left(\mathcal{S}_{11}, \mathcal{H}_{11}\right)=\Lambda_{-}\left(\mathcal{B}_{\mathcal{S}}^{c}, \mathcal{B}_{\mathcal{H}}^{c}\right)$.

In the following, we will use the linear space $\mathbb{C}^{n, n} \times \mathbb{C}^{n, n}$ endowed with the norm

$$
\|(X, Y)\|=\max \left\{\|X\|_{2},\|Y\|_{2}\right\} .
$$

THEOREM 5.3. Let $\alpha \mathcal{S}-\beta \mathcal{H}$ be a regular skew-Hamiltonian/Hamiltonian matrix pencil with neither infinite nor purely imaginary eigenvalues. Let $\mathcal{P}_{V}^{-}$be the orthogonal basis of the deflating subspace of $\alpha \mathcal{S}-\beta \mathcal{H}$ corresponding to $\Lambda_{-}(\mathcal{S}, \mathcal{H})$, and let $\hat{\mathcal{P}}_{V}^{-}$be the perturbation of $\mathcal{P}_{V}^{-}$obtained by Algorithm 2 in finite precision arithmetic. Denote by $\Theta \in \mathbb{C}^{n, n}$ the diagonal matrix of canonical angles between $\mathcal{P}_{V}^{-}$and $\hat{\mathcal{P}}_{V}^{-}$.

Using the structured Schur form of the extended skew-Hamiltonian/Hamiltonian matrix pencil $\alpha \mathcal{B}_{\mathcal{S}}^{c}-\beta \mathcal{B}_{\mathcal{H}}^{c}$ (as in (2.17) and (2.22)) given by (5.17), define $\delta$ by

$$
\delta=\min _{Y \in \mathbb{C}^{2 n, 2 n} \backslash\{0\}} \frac{\left\|\left(\mathcal{H}_{11}^{H} Y+Y^{H} \mathcal{H}_{11}, \mathcal{S}_{11}^{H} Y-Y^{H} \mathcal{S}_{11}\right)\right\|}{\|Y\|_{2}} .
$$

If

$$
8\left\|\left(\mathcal{E}_{\mathcal{S}}, \mathcal{E}_{\mathcal{H}}\right)\right\|\left(\delta+\left\|\left(\mathcal{S}_{12}, \mathcal{H}_{12}\right)\right\|\right)<\delta^{2},
$$


then

$$
\|\Theta\|_{2}<c_{b} \frac{\left\|\left(\mathcal{E}_{\mathcal{S}}, \mathcal{E}_{\mathcal{H}}\right)\right\|}{\delta}<c_{b} \varepsilon \frac{\left\|\left(c_{\mathcal{S}} \mathcal{S}, c_{\mathcal{H}} \mathcal{H}\right)\right\|}{\delta}
$$

where $c_{\mathcal{S}}$ and $c_{\mathcal{H}}$ are the modest constants in (5.3)-(5.4) and $c_{b}=8(\sqrt{10}+4) /(\sqrt{10}+2) \approx 11.1$.

Proof. Let $\alpha \hat{\mathcal{R}}_{\mathcal{S}}-\beta \hat{\mathcal{R}}_{\mathcal{H}}, \hat{\mathcal{Q}}$ be the output of Step 2 in Algorithm 2 in finite precision arithmetic, where $\hat{\mathcal{B}}_{\mathcal{S}}^{c}, \hat{\mathcal{B}}_{\mathcal{H}}^{c}$ satisfy (5.1) and (5.2). Let $\tilde{\mathcal{Q}}$ be the unitary matrix computed by Algorithm 2 in exact arithmetic such that

$$
\begin{aligned}
\mathcal{J} \tilde{\mathcal{Q}}^{H} \mathcal{J}^{T}\left(\alpha \mathcal{B}_{\mathcal{S}}^{c}-\beta \mathcal{B}_{\mathcal{H}}^{c}\right) \tilde{\mathcal{Q}} & =\alpha \tilde{\mathcal{R}}_{\mathcal{S}}-\beta \tilde{\mathcal{R}}_{\mathcal{H}} \\
& =\alpha\left[\begin{array}{cc}
\tilde{\mathcal{S}}_{11} & \tilde{\mathcal{S}}_{12} \\
0 & \tilde{\mathcal{S}}_{11}^{H}
\end{array}\right]-\beta\left[\begin{array}{cc}
\tilde{\mathcal{H}}_{11} & \tilde{\mathcal{H}}_{12} \\
0 & -\tilde{\mathcal{H}}_{11}^{H}
\end{array}\right],
\end{aligned}
$$

with $\Lambda\left(\tilde{\mathcal{S}}_{11}, \tilde{\mathcal{H}}_{11}\right)=\Lambda_{-}\left(\mathcal{B}_{\mathcal{S}}^{c}, \mathcal{B}_{\mathcal{H}}^{c}\right)$. Since (5.17) is another structured Schur form with the same eigenvalue ordering, there exists a unitary diagonal matrix $\mathcal{G}=\operatorname{diag}\left(G_{1}, G_{2}\right)$ such that $\mathcal{Q}=\tilde{\mathcal{Q}} \mathcal{G}$. Therefore, we have

$$
\left\|\left(\tilde{\mathcal{S}}_{12}, \tilde{\mathcal{H}}_{12}\right)\right\|=\left\|\left(\mathcal{S}_{12}, \mathcal{H}_{12}\right)\right\|,
$$

and for $\delta$ given in (5.18) we also have

$$
\delta=\min _{Y \in \mathbb{C}^{2 n, 2 n} \backslash\{0\}} \frac{\left\|\left(\tilde{\mathcal{H}}_{11}^{H} Y+Y^{H} \tilde{\mathcal{H}}_{11}, \tilde{\mathcal{S}}_{11}^{H} Y-Y^{H} \tilde{\mathcal{S}}_{11}\right)\right\|}{\|Y\|_{2}} .
$$

Let

$$
\tilde{\mathcal{E}}_{\mathcal{S}}:=\mathcal{J} \tilde{\mathcal{Q}}^{H} \mathcal{J}^{T} \mathcal{E}_{\mathcal{S}} \tilde{\mathcal{Q}}=:\left[\begin{array}{ll}
\mathcal{E}_{11} & \mathcal{E}_{12} \\
\mathcal{E}_{21} & \mathcal{E}_{11}^{H}
\end{array}\right], \quad \tilde{\mathcal{E}}_{\mathcal{H}}:=\mathcal{J} \tilde{\mathcal{Q}}^{H} \mathcal{J}^{T} \mathcal{E}_{\mathcal{H}} \tilde{\mathcal{Q}}=:\left[\begin{array}{cc}
\mathcal{F}_{11} & \mathcal{F}_{12} \\
\mathcal{F}_{21} & -\mathcal{F}_{11}^{H}
\end{array}\right]
$$

and set $\gamma=\left\|\left(\mathcal{E}_{21}, \mathcal{F}_{21}\right)\right\|, \eta=\left\|\left(\tilde{\mathcal{S}}_{12}+\mathcal{E}_{12}, \tilde{\mathcal{H}}_{12}+\mathcal{F}_{12}\right)\right\|$ and $\tilde{\delta}=\delta-2\left\|\left(\mathcal{E}_{11}, \mathcal{F}_{11}\right)\right\|$. Since we have $\left\|\left(\tilde{\mathcal{E}}_{\mathcal{S}}, \tilde{\mathcal{E}}_{\mathcal{H}}\right)\right\|=\left\|\left(\mathcal{E}_{\mathcal{S}}, \mathcal{E}_{\mathcal{H}}\right)\right\|$, condition (5.19) implies that

$$
\tilde{\delta} \geq \delta-2\left\|\left(\mathcal{E}_{\mathcal{S}}, \mathcal{E}_{\mathcal{H}}\right)\right\|>\frac{3}{4} \delta
$$

and clearly,

$$
4\left\|\left(\mathcal{E}_{\mathcal{S}}, \mathcal{E}_{\mathcal{H}}\right)\right\|\left\|\left(\mathcal{S}_{12}, \mathcal{H}_{12}\right)\right\|<\delta^{2}-4 \delta\left\|\left(\mathcal{E}_{\mathcal{S}}, \mathcal{E}_{\mathcal{H}}\right)\right\|
$$

Hence

$$
\begin{aligned}
\frac{\gamma \eta}{\tilde{\delta}^{2}} \leq & \frac{\left\|\left(\mathcal{E}_{\mathcal{S}}, \mathcal{E}_{\mathcal{H}}\right)\right\|\left\{\left\|\left(\tilde{\mathcal{S}}_{12}, \tilde{\mathcal{H}}_{12}\right)\right\|+\left\|\left(\mathcal{E}_{\mathcal{S}}, \mathcal{E}_{\mathcal{H}}\right)\right\|\right\}}{\left(\delta-2\left\|\left(\mathcal{E}_{\mathcal{S}}, \mathcal{E}_{\mathcal{H}}\right)\right\|\right)^{2}} \\
& <\frac{\left\|\left(\mathcal{E}_{\mathcal{S}}, \mathcal{E}_{\mathcal{H}}\right)\right\|^{2}+\left(\delta^{2}-4 \delta\left\|\left(\mathcal{E}_{\mathcal{S}}, \mathcal{E}_{\mathcal{H}}\right)\right\|\right) / 4}{\left(\delta-2\left\|\left(\mathcal{E}_{\mathcal{S}}, \mathcal{E}_{\mathcal{H}}\right)\right\|\right)^{2}}=\frac{1}{4} .
\end{aligned}
$$

Following the perturbation analysis for a formal product of matrices in [9], it can be shown that there exists a unitary matrix

$$
\mathcal{W}=\left[\begin{array}{cc}
\left(I+W^{H} W\right)^{-\frac{1}{2}} & -W^{H}\left(I+W W^{H}\right)^{-\frac{1}{2}} \\
W\left(I+W^{H} W\right)^{-\frac{1}{2}} & \left(I+W W^{H}\right)^{-\frac{1}{2}}
\end{array}\right]
$$

with

$$
\|W\|_{2}<2 \frac{\gamma}{\tilde{\delta}}<\frac{8}{3} \frac{\gamma}{\delta}<\frac{1}{3}
$$


such that

$$
\mathcal{J}(\tilde{\mathcal{Q}} \mathcal{W})^{H} \mathcal{J}^{T}\left(\alpha \hat{\mathcal{B}}_{\mathcal{S}}^{c}-\beta \hat{\mathcal{B}}_{\mathcal{H}}^{c}\right)(\tilde{\mathcal{Q}} \mathcal{W})
$$

is another structured Schur form of the perturbed matrix pencil. Since there are neither infinite nor purely imaginary eigenvalues, (5.16) implies that $\hat{\mathcal{Q}}^{H} \tilde{\mathcal{Q}} \mathcal{W}$ is unitary block diagonal.

Without loss of generality we may take $\hat{\mathcal{Q}}=\tilde{\mathcal{Q}} \mathcal{W}$. If $\mathcal{X}_{c}$ is as in $(2.11)$ and $\mathcal{X}_{c} \tilde{\mathcal{Q}}=\left[\begin{array}{ll}\mathcal{Q}_{11} & \mathcal{Q}_{12} \\ \mathcal{Q}_{21} & \mathcal{Q}_{22}\end{array}\right]$, then it follows from Theorem 3.1 that $\mathcal{P}_{V}^{-}=$range $\mathcal{Q}_{11}$. Clearly $\hat{\mathcal{P}}_{V}^{-}=\operatorname{range}\left\{\left(\mathcal{Q}_{11}+\mathcal{Q}_{12} W\right)(I+\right.$ $\left.\left.W^{H} W\right)^{-\frac{1}{2}}\right\}$. The upper bound (5.20) can then be derived from (5.21), by using the same argument as in the proof of Theorem 4.4 in [6].

If $\mathcal{S}$ is given in factored form, then we obtain a similar result. In this case, by using Algorithm 1 we compute a unitary matrix $\hat{\mathcal{Q}}$ and a unitary symplectic matrix $\hat{\mathcal{U}}$ such that

$$
\begin{array}{r}
\hat{\mathcal{U}}^{H} \hat{\mathcal{B}}_{\mathcal{Z}}^{c} \hat{\mathcal{Q}}=\hat{\mathcal{R}}_{\mathcal{Z}}=:\left[\begin{array}{cc}
\hat{\mathcal{Z}}_{11} & \hat{\mathcal{Z}}_{12} \\
0 & \hat{\mathcal{Z}}_{22}
\end{array}\right], \\
\mathcal{J} \hat{\mathcal{Q}}^{H} \mathcal{J}^{T} \hat{\mathcal{B}}_{\mathcal{H}}^{c} \hat{\mathcal{Q}}=\hat{\mathcal{R}}_{\mathcal{H}}=:\left[\begin{array}{cc}
\hat{\mathcal{H}}_{11} & \hat{\mathcal{H}}_{12} \\
0 & -\hat{\mathcal{H}}_{11}^{H}
\end{array}\right],
\end{array}
$$

where $\hat{\mathcal{B}}_{\mathcal{Z}}^{c}$ and $\hat{\mathcal{B}}_{\mathcal{H}}^{c}$ are defined in (5.10) and (5.11), and $\Lambda\left(\hat{\mathcal{Z}}_{22}^{H} \hat{\mathcal{Z}}_{11}, \hat{\mathcal{H}}_{11}\right)=\Lambda_{-}\left(\hat{\mathcal{B}}_{\mathcal{S}}^{c}, \hat{\mathcal{B}}_{\mathcal{H}}^{c}\right)$, where $\hat{\mathcal{B}}_{\mathcal{S}}^{c}=\mathcal{J}\left(\hat{\mathcal{B}}_{\mathcal{Z}}^{c}\right)^{H} \mathcal{J}^{T} \hat{\mathcal{B}}_{\mathcal{Z}}^{c}$.

Analogous to Theorem 2.7, if $\alpha \mathcal{S}-\beta \mathcal{H}$ has no purely imaginary eigenvalues, then there exist unitary matrices $\mathcal{Q}_{1}, \mathcal{Q}_{2}$ and unitary symplectic matrices $\mathcal{U}_{1}, \mathcal{U}_{2}$ such that

$$
\mathcal{U}_{1}^{H} \mathcal{Z} \mathcal{Q}_{1}=\left[\begin{array}{cc}
Z_{11}^{-} & Z_{12}^{-} \\
0 & Z_{22}^{-}
\end{array}\right], \quad \mathcal{J} \mathcal{Q}_{1}^{H} \mathcal{J}^{T} \mathcal{H} \mathcal{Q}_{1}=\left[\begin{array}{cc}
H_{11}^{-} & H_{12}^{-} \\
0 & -\left(H_{11}^{-}\right)^{H}
\end{array}\right]
$$

with $\Lambda\left(\left(Z_{22}^{-}\right)^{H} Z_{11}^{-}, H_{11}^{-}\right)=\Lambda_{-}(\mathcal{S}, \mathcal{H})$, and

$$
\mathcal{U}_{2}^{H} \mathcal{Z} \mathcal{Q}_{2}=\left[\begin{array}{cc}
Z_{11}^{+} & Z_{12}^{+} \\
0 & Z_{22}^{+}
\end{array}\right], \quad \mathcal{J} \mathcal{Q}_{2}^{H} \mathcal{J}^{T} \mathcal{H} \mathcal{Q}_{2}=\left[\begin{array}{cc}
H_{11}^{+} & H_{12}^{+} \\
0 & -\left(H_{11}^{+}\right)^{H}
\end{array}\right],
$$

with $\Lambda\left(\left(Z_{22}^{+}\right)^{H} Z_{11}^{+}, H_{11}^{+}\right)=\Lambda_{+}(\mathcal{S}, \mathcal{H})$, respectively. Set

$$
\mathcal{Q}=\mathcal{X}_{c}^{H} \operatorname{diag}\left(\mathcal{Q}_{1}, \overline{\mathcal{Q}}_{2}\right) \mathcal{P}, \quad \mathcal{U}=\mathcal{X}_{c}^{H} \operatorname{diag}\left(\mathcal{U}_{1}, \overline{\mathcal{U}}_{2}\right) \mathcal{P},
$$

where $\mathcal{P}$ and $\mathcal{X}_{c}$ are as in (2.10) and (2.11). Then $\mathcal{Q}$ is unitary and $\mathcal{U} \in \mathbb{U S}_{4 n}$, and a simple calculation yields

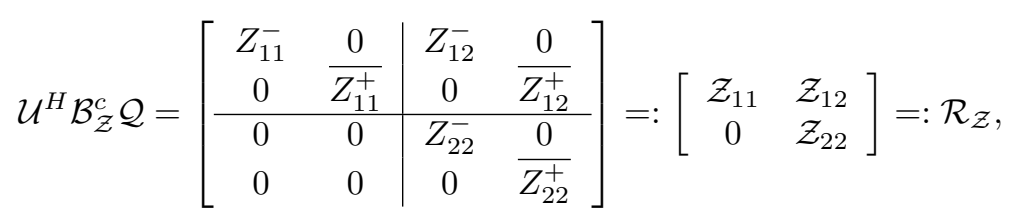

$(5.24) \mathcal{J} \mathcal{Q}^{H} \mathcal{J}^{T} \mathcal{B}_{\mathcal{H}}^{c} \mathcal{Q}=\left[\begin{array}{cc|cc}H_{11}^{-} & 0 & H_{12}^{-} & 0 \\ 0 & -\overline{H_{11}^{+}} & 0 & -\overline{H_{12}^{+}} \\ \hline 0 & 0 & -\left(H_{11}^{-}\right)^{H} & 0 \\ 0 & 0 & 0 & \left(\overline{H_{11}^{+}}\right)^{H}\end{array}\right]=:\left[\begin{array}{cc}\mathcal{H}_{11} & \mathcal{H}_{12} \\ 0 & -\mathcal{H}_{11}^{H}\end{array}\right]=: \mathcal{R}_{\mathcal{H}}$

This leads to the structured Schur form of the extended skew-Hamiltonian/Hamiltonian matrix pencil $\alpha \mathcal{J}\left(\mathcal{B}_{\mathcal{Z}}^{c}\right)^{H} \mathcal{J}^{T} \mathcal{B}_{\mathcal{Z}}^{c}-\beta \mathcal{B}_{\mathcal{H}}^{c}$ with $\Lambda\left(\mathcal{Z}_{22}^{H} \mathcal{Z}_{11}, \mathcal{H}_{11}\right)=\Lambda_{-}\left(\mathcal{B}_{\mathcal{S}}^{c}, \mathcal{B}_{\mathcal{H}}^{c}\right)$.

THEOREM 5.4. Consider the regular skew-Hamiltonian/Hamiltonian matrix pencil $\alpha \mathcal{S}-\beta \mathcal{H}$ with nonsingular, $\mathcal{J}$-definite skew-Hamiltonian part $\mathcal{S}=\mathcal{J Z}^{H} \mathcal{J}^{T} \mathcal{Z}$. Suppose that $\alpha \mathcal{S}-\beta \mathcal{H}$ has no eigenvalue with zero real part. Let the extended skew-Hamiltonian and Hamiltonian matrix $\mathcal{B}_{\mathcal{Z}}^{c}$ 
and $\mathcal{B}_{\mathcal{H}}^{c}$ be as in (2.16) and (2.22), respectively with structured triangular form given by (5.23) and (5.24). Define $\delta_{p}$ as

$$
\delta_{p}=\min _{(X, Y) \in \mathbb{C}^{2 n, 2 n} \times \mathbb{C}^{2 n, 2 n} \backslash\{(0,0)\}} \frac{\left\|\left(\mathcal{H}_{11}^{H} Y+Y^{H} \mathcal{H}_{11}, X \mathcal{Z}_{11}-\mathcal{Z}_{22} Y\right)\right\|}{\|(X, Y)\|_{2}} .
$$

Define errors $\mathcal{E}_{\mathcal{Z}}$ and $\mathcal{E}_{\mathcal{H}}$ by (5.10) and (5.11). Let $\mathcal{P}_{V}^{-}, \mathcal{P}_{U}^{-}, \hat{\mathcal{P}}_{V}^{-}$and $\hat{\mathcal{P}}_{U}^{-}$be the deflating subspaces computed by Algorithm 1 in exact and finite precision arithmetic, respectively. Denote by $\Theta_{V}, \Theta_{U} \in$ $\mathbb{C}^{n, n}$ the diagonal matrices of canonical angles between $\mathcal{P}_{V}^{-}$and $\hat{\mathcal{P}}_{V}^{-}, \mathcal{P}_{U}^{-}$and $\hat{\mathcal{P}}_{U}^{-}$, respectively.

If

$$
8\left\|\left(\mathcal{E}_{\mathcal{Z}}, \mathcal{E}_{\mathcal{H}}\right)\right\|\left(\delta_{p}+\left\|\left(\mathcal{Z}_{12}, \mathcal{H}_{12}\right)\right\|\right)<\delta_{p}^{2}
$$

then

$$
\left\|\Theta_{V}\right\|_{2},\left\|\Theta_{U}\right\|_{2}<c_{b} \frac{\left\|\left(\mathcal{E}_{\mathcal{Z}}, \mathcal{E}_{\mathcal{H}}\right)\right\|}{\delta_{p}}<c_{b} \varepsilon \frac{\left\|\left(c_{\mathcal{Z}} \mathcal{Z}, c_{\mathcal{H}} \mathcal{H}\right)\right\|}{\delta_{p}}
$$

with $c_{b}$ as in Theorem 5.3.

Proof. The proof is analogous to the proof of Theorem 5.3. $\quad \square$

It follows that the described numerical algorithms are numerically backwards stable. These algorithms can also be used to compute deflating subspaces which contain eigenvectors associated with infinite or purely imaginary eigenvalues. By Theorem 3.1 we get partial information also in these cases, but we face the difficulty that the desired deflating subspace may not be unique or may not exist. (See the recent analysis for Hamiltonian matrices [29]).

6. Conclusion. We have presented numerical procedures for the computation of structured Schur forms, eigenvalues, and deflating subspaces of matrix pencils with matrices having a Hamiltonian and/or skew-Hamiltonian structure. These methods generalize the recently developed methods for Hamiltonian matrices which use an extended, double dimension Hamiltonian matrix that always has a Hamiltonian Schur form.

The algorithms circumvent problems with skew-Hamiltonian/Hamiltonian matrix pencils that lack a structured Schur form by embedding them in extended matrix pencils that always admit a structured Schur form. For the extended matrix pencils, the algorithms use structure preserving unitary matrix computations and are strongly backwards stable, i.e., they compute the exact structured Schur form of a nearby matrix pencil with the same structure. Such structured Schur forms can always be computed regardless of the regularity of the original matrix pencil.

It is still somewhat unsatisfactory that the algorithms do not efficiently exploit the micro structures of the extended matrix pencils, as for example in the matrix $\mathcal{B}_{\mathcal{N}}^{c}$ in (2.12). How best to use these micro structures is still an open question.

Practical implementation and numerical experiments are in progress and will be reported elsewhere. For detailed algorithms and implementation issues see [4].

Acknowledgments. We gratefully acknowledge Daniel Kressner for his assistance implementing and testing experimental versions of parts of the algorithms discussed here.

\section{REFERENCES}

[1] G. Ammar, P. Benner, and V. Mehrmann, A multishift algorithm for the numerical solution of algebraic Riccati equations, Electr. Trans. Num. Anal., 1 (1993), pp. 33-48.

[2] G. Ammar and V. Mehrmann, On Hamiltonian and symplectic Hessenberg forms, Linear Algebra Appl., 149 (1991), pp. 55-72.

[3] P. Benner, R. Byers, H. Fassbender, V. Mehrmann, and D. Watkins, Cholesky-like factorizations of skew-symmetric matrices, Electr. Trans. Num. Anal., 11 (2000), pp. 85-93.

[4] P. Benner, R. Byers, V. Mehrmann, and H. Xu, Numerical computation of deflating subspaces of embedded Hamiltonian pencils, Tech. Report SFB393/99-15, Fakultät für Mathematik, TU Chemnitz, 09107 Chemnitz, FRG, 1999. Available from http://www.tu-chemnitz.de/sfb393/sfb99pr.html. 
[5] - Numerical methods for linear-quadratic and $H_{\infty}$ control problems, in Dynamical Systems, Control, Coding, Computer Vision: New Trends, Interfaces, and Interplay, G. Picci and D. Gilliam, eds., vol. 25 of Progress in Systems and Control Theory, Birkhäuser, Basel, 1999, pp. 203-222.

[6] P. Benner, V. Mehrmann, And H. Xu, A new method for computing the stable invariant subspace of a real Hamiltonian matrix, J. Comput. Appl. Math., 86 (1997), pp. 17-43.

[7] _ - A numerically stable, structure preserving method for computing the eigenvalues of real Hamiltonian or symplectic pencils, Numer. Math., 78 (1998), pp. 329-358.

[8] - A note on the numerical solution of complex Hamiltonian and skew-Hamiltonian eigenvalue problems, Electr. Trans. Num. Anal., 8 (1999), pp. 115-126.

[9] - Perturbation analysis for the eigenvalue problem of a formal product of matrices, Berichte aus der Technomathematik, Report 00-01, FB3 - Mathematik und Informatik, Universität Bremen, 28334 Bremen (Germany), Jan. 2000. Available from http://www.math.uni-bremen.de/zetem/berichte.html.

[10] A. Bojanczyk, G. Golub, and P. Van Dooren, The periodic Schur decomposition; algorithms and applications, in Proc. SPIE Conference, vol. 1770, 1992, pp. 31-42.

[11] A. Bunse-Gerstner, Matrix factorization for symplectic QR-like methods, Linear Algebra Appl., 83 (1986), pp. $49-77$.

[12] A. Bunse-Gerstner, R. Byers, and V. Mehrmann, Numerical methods for algebraic Riccati equations, in Proc. Workshop on the Riccati Equation in Control, Systems, and Signals, S. Bittanti, ed., Como, Italy, 1989, pp. 107-116.

[13] R. BYERS, Hamiltonian and Symplectic Algorithms for the Algebraic Riccati Equation, PhD thesis, Cornell University, Dept. Comp. Sci., Ithaca, NY, 1983.

[14] — A Hamiltonian QR-algorithm, SIAM J. Sci. Statist. Comput., 7 (1986), pp. 212-229.

[15] H. Fassbender, D. Mackey, N. Mackey, and H. Xu, Hamiltonian square roots of skew-Hamiltonian matrices, Linear Algebra Appl., 287 (1998), pp. 125-159.

[16] F. Gantmacher, Theory of Matrices, vol. 1, Chelsea, New York, 1959.

[17] G. Golub and C. Van Loan, Matrix Computations, Johns Hopkins University Press, Baltimore, third ed., 1996.

[18] M. Green and D. Limebeer, Linear Robust Control, Prentice-Hall, Englewood Cliffs, NJ, 1995.

[19] J. Hench and A. Laub, Numerical solution of the discrete-time periodic Riccati equation, IEEE Trans. Automat. Control, 39 (1994), pp. 1197-1210.

[20] P. Lancaster, Strongly stable gyroscopic systems, Electr. J. Linear Algebra, 5 (1999), pp. 53-66.

[21] P. Lancaster and M. Tismenetsky, The Theory of Matrices, Academic Press, Orlando, 2nd ed., 1985.

[22] A. LAUB, Invariant subspace methods for the numerical solution of Riccati equations, in The Riccati Equation, S. Bittanti, A. Laub, and J. Willems, eds., Springer-Verlag, Berlin, 1991, pp. 163-196.

[23] W.-W. Lin And T.-C. Ho, On Schur type decompositions for Hamiltonian and symplectic pencils, tech. report, Institute of Applied Mathematics, National Tsing Hua University, Taiwan, 1990.

[24] W.-W. Lin, V. Mehrmann, and H. XU, Canonical forms for Hamiltonian and symplectic matrices and pencils, Linear Algebra Appl., 301-303 (1999), pp. 469-533.

[25] C. MenL, Compatible Lie and Jordan algebras and applications to structured matrices and pencils, dissertation, Fakultät für Mathematik, TU Chemnitz, 09107 Chemnitz (FRG), 1998.

[26] - Condensed forms for skew-Hamiltonian/Hamiltonian pencils, SIAM J. Matrix Anal. Appl., 21 (1999), pp. $454-476$

[27] V. Menrmann, The Autonomous Linear Quadratic Control Problem, Theory and Numerical Solution, no. 163 in Lecture Notes in Control and Information Sciences, Springer-Verlag, Heidelberg, July 1991.

[28] V. Mehrmann AND D. WATKIns, Structure-preserving methods for computing eigenpairs of large sparse skew-Hamiltonian/Hamiltonian pencils, SIAM J. Sci. Comput., (2001). To appear. Available from http://www.tu-chemnitz.de/sfb393/sfb00pr.html.

[29] V. Mehrmann and H. XU, Lagrangian invariant subspaces of Hamiltonian matrices, Tech. Report SFB393/98-25, Fakultät für Mathematik, TU Chemnitz, 09107 Chemnitz, FRG, 1998. Available from http://www.tu-chemnitz.de/sfb393/sfb98pr.html.

[30] J. Olson, H. Jensen, And P. JøRGensen, Solution of large matrix equations which occur in response theory, J. Comput. Phys., 74 (1988), pp. 265-282.

[31] C. Paige and C. Van Loan, A Schur decomposition for Hamiltonian matrices, Linear Algebra Appl., 14 (1981), pp. 11-32.

[32] R. PATEL, On computing the eigenvalues of a symplectic pencil, Linear Algebra Appl., 188/189 (1993), pp. 591611.

[33] P. Petkov, N. Christov, and M. Konstantinov, Computational Methods for Linear Control Systems, Prentice-Hall, Hertfordshire, UK, 1991.

[34] A.-M. SÄNDIG AND W. Wendland, Asymptotic expansions of elastic fields in domains with boundary and structural singularities, in Boundary element topics (Stuttgart, 1995), Springer, Berlin, 1997, pp. 419-444.

[35] V. Sima, Algorithms for Linear-Quadratic Optimization, vol. 200 of Pure and Applied Mathematics, Marcel Dekker, Inc., New York, NY, 1996.

[36] G. Stewart and J.-G. Sun, Matrix Perturbation Theory, Academic Press, New York, 1990.

[37] P. VAn Dooren, A generalized eigenvalue approach for solving Riccati equations, SIAM J. Sci. Statist. Comput., 2 (1981), pp. 121-135.

[38] C. VAN LOAN, A symplectic method for approximating all the eigenvalues of a Hamiltonian matrix, Linear Algebra Appl., 61 (1984), pp. 233-251. 
[39] K. Zhou, J. Doyle, and K. Glover, Robust and Optimal Control, Prentice-Hall, Upper Saddle River, NJ, 1995. 